\title{
Timing and low-level rf system for an x-ray laser
}

\author{
Yuji Otake, ${ }^{1}$ Hirokazu Maesaka, ${ }^{1}$ Shinich Matsubara, ${ }^{3}$ \\ Naoyasu Hosoda, ${ }^{2}$ and Takashi Ohshima ${ }^{1}$ \\ ${ }^{1}$ XFEL Research and Development Division, RIKEN SPring-8 Center, \\ RIKEN 1-1-1 Kouto, Sayo-cho, Sayo-gun, Hyogo 679-5148, Japan \\ ${ }^{2}$ Innovative Light Source Division, RIKEN SPring-8 Center, RIKEN 1-1-1 Kouto, \\ Sayo-cho, Sayo-gun, Hyogo 679-5148, Japan \\ ${ }^{3}$ XFEL Utilization Division, JASRI 1-1-1 Kouto, Sayo-cho, Sayo-gun, Hyogo 679-5198, Japan
}

(Received 3 July 2015; published 19 February 2016)

\begin{abstract}
An x-ray free-electron laser (XFEL), SACLA, designed to open up new science, was constructed for generating coherent $\mathrm{x}$ rays with a peak power of more than $10 \mathrm{GW}$ and a very short pulse of below $30 \mathrm{fs}$. This feature demands a very highly short-term temporal stability of less than $50 \mathrm{fs}$ to the acceleration rf field of SACLA. For this reason, we developed a timing and low-level rf (LLRF) system for SACLA based on that of the SPring8 compact SASE source (SCSS) test accelerator for verifying the feasibility of an XFEL. The performance of the system using the in-phase and quadrature rf manipulation method was improved from SCSS's system. Since the facility length of SACLA is $700 \mathrm{~m}$, which is 10 times longer than that of the SCSS test accelerator, a phase-stabilized optical-fiber system designed to transmit time standard rf signals with low loss was also developed and deployed. This optical-fiber system equips fiber optical-length feedback control in order to mitigate environmental effects, such as temperature and humidity changes. On the other hand, the demanded maximum rf temporal stability is less than $50 \mathrm{fs}$, which is almost 10 times smaller than that of the SCSS test accelerator. Hence, reducing electric noise and increasing the temperature stability around timing and LLRF instruments were necessary and realized with a very low-noise power supply and a hemathermal 19-inch enclosure. The short-term temporal performance of the timing LLRF system finally attained a temporal stability of less than $13.6 \mathrm{fs}$ in rms measured by a beam arrival-time measurement. This stability greatly helps to achieve the stable x-ray lasing of SACLA for routine operation during user experiments.
\end{abstract}

DOI: 10.1103/PhysRevAccelBeams.19.022001

\section{INTRODUCTION}

An X-ray laser, which is generated by a free-electron laser (XFEL) machine [1], using the self-amplified spontaneous emission (SASE) method, is a dream light source used to explore uncharted science. One of the important features of XFEL is a short pulse width of below $30 \mathrm{fs}$. By using this feature, a Ti-sapphire laser pump and x-ray laser probe experiment (a pump-probe experiment), which demands a temporally ultrastable $\mathrm{x}$-ray laser, is one of the most prominent experiments at XFEL. The generation mechanism of SASE involves a nonlinear interaction between an electron beam and the periodically and sinusoidally changed magnetic field of an undulator. In this interaction, a very high electron density corresponding to more than several kiloamperes is necessary [2]. In the present particle accelerator technology, the direct generation of this kiloampere order electron beam from an electron gun is impossible. Therefore,

Published by the American Physical Society under the terms of the Creative Commons Attribution 3.0 License. Further distribution of this work must maintain attribution to the author(s) and the published article's title, journal citation, and DOI. an electron bunch-length compression method is needed in order to form a high-peak current beam with a short pulse width from a low-peak current beam with a long pulse width. For example, in the case of the SPring-8 Angstrom compact laser (SACLA) [3] in order to realize an $\mathrm{x}$-ray laser, there are two bunch-compression methods. One is a velocity bunchcompression process using multisubharmonic bunchers in an injector; the other is a magnetic bunching process involving bunch compressors (BCs) composed of a chicane with four bending magnets. When employing the bunch-compression methods, we use electron acceleration at the off-crest part of an rf field in a cavity. In the velocity bunching process, the velocity modulation along an electron bunch given with the cavity directly converts to its bunch length (an electron position within the bunch) through an electron densitymodulation process for a drift space. Similarly, in the magnetic compressing process, the energy chirp along the electron bunch given with a cavity just upstream of the $\mathrm{BC}$ makes the bunch length short according to the R56 parameter in the BC. Therefore, the peak current value of the electron bunch sensitively changes due to its energy variation.

For example, the velocity bunching process is expressed by [4] 


$$
X=\frac{\theta+\omega\left(t_{1}-t_{2}\right)}{\sin \left(\omega t_{1}+\phi\right)}
$$

where $X$ is the bunching parameter, written as $X=$ $\left(V_{1} / 2 V_{0}\right) \theta$. The $X$ is like a bunch strength parameter showing the relation between the initial peak-current value and the final bunched-up peak-current value. Here, $V_{0}$ and $V_{1}$ are the beam acceleration voltage of an electron gun and the electron velocity-modulation $\mathrm{rf}$ voltage at a buncher, respectively; $\theta$ is the drift angle, expressed by $\theta=\omega s / v_{0}$; $v_{0}$ is the initial speed of the electron generated with the electron gun; $s$ is the position based on the coordinates of an electron motion direction; $\omega$ is the angular frequency of the bunching rf; $t_{1}$ is the initial time at the buncher; and $t_{2}$ is the drift time of the electron after the buncher. This equation is a linearly approximated expression.

From the discussion mentioned above and Ref. [4], if the bunching rf phase $\phi$ changes, the longitudinal bunch-peak position shifts. Then, the initial phase of the beam to an acceleration rf, which gives an energy chirp along the bunch for the $\mathrm{BC}$, also varies. This beam-phase change finally connects to the bunch-length variation in accordance with the argument given below.

Next, the magnetic bunching process is expressed by [5]

$$
\frac{\Delta \sigma_{s}}{\sigma_{s}} \simeq-C \frac{\Delta \phi}{\phi}
$$

when the bunching factor of $C$ has the relation $C \gg 1$. In this case, $\phi$ is the rf phase of the cavity before the $\mathrm{BC}$ in order to give an energy chirp along the electron bunch; $\Delta \phi$ is the change of $\phi ; \sigma_{s}$ is the bunch length after the $\mathrm{BC}$; and $\Delta \sigma_{s}$ is the change of $\sigma_{s}$. Both the bunching processes directly connected to a bunch length have big effects due to rf phase changes of the cavities, since the phase variations correspond to an energy variation along the electron bunch. These effects are understandable from Eqs. (1) and (2).

SACLA, as shown in Fig. 1, has been constructed at the SPring- 8 site and comprises an $8-\mathrm{GeV}$ linear accelerator, which is $400 \mathrm{~m}$ long, and having 18 in-vacuum undulators, each being $5 \mathrm{~m}$ long. The accelerator consists of an injector including 238, 476, and $1428 \mathrm{MHz}$ ( $L$-band) multisubharmonic bunchers and a main accelerator using 2856 ( $S$-band) and $5712 \mathrm{MHz}$ ( $C$-band) traveling-wave accelerating structures. Based on the bunch-compression methods, as mentioned above, the required temporal stabilities at the cavities along SACLA are tabulated in Table I [6]. These values in the table were calculated under the condition where the peak current variation of the electron beam is $10 \%$ in rms. This $10 \%$ variation conducts an $\mathrm{x}$-ray laser intensity change of $10 \%$ in rms [6]. The laser intensity fluctuation almost corresponds to the natural intensity variation of the SASE process in the undulator. In the case of SACLA, the bunch-compression ratio through the velocity bunching and magnetic bunching processes from the injector to BC1-3 is more than 3000 . This extremely large bunch compression is one of the causes of the tight temporal tolerance tabulated in Table I.

When we designed a low-level rf (LLRF) system in order to drive a klystron as a high-power if source for electron acceleration during the construction stage of SACLA, the first priority was how to realize the demanded temporal stabilities. At this point, a short-period temporal jitter of about $50 \mathrm{fs}$ in rms and a temporal drift of over $300 \mathrm{fs}$ in peak-to-peak (P-P) for $12 \mathrm{~h}$ of an electron beam were already observed by using our developed LLRF system [7] of the SPring8 compact SASE source (SCSS) test accelerator [8]. The environmental condition changes, such as the temperature variation, mainly affected the drift of $300 \mathrm{fs}$, which corresponds to a phase of about $1^{\circ}$ at $5712 \mathrm{MHz}$, being the main-acceleration rf frequency of SACLA. Furthermore, we occasionally observed a further temporal drift value of over 1 ps (P-P), when a large environmental temperature change of over several kelvins occurred outside of an enclosure in order to install LLRF instruments. Since the purpose of the SCSS test accelerator was to check the feasibility of SACLA, a newly designed LLRF system for SACLA should be based on the LLRF

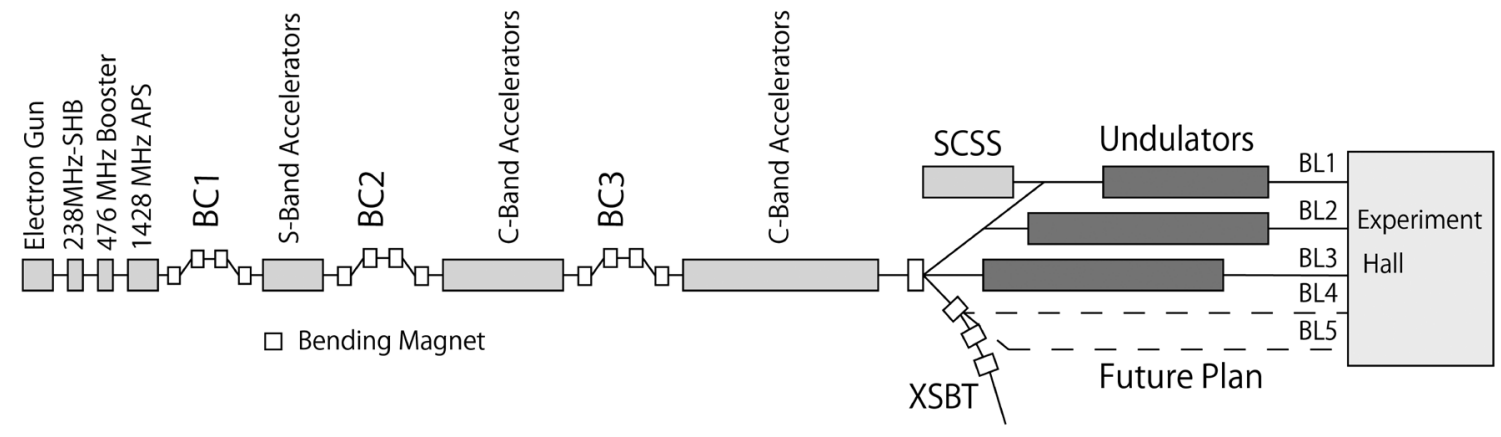

FIG. 1. Configuration of SACLA. From the left side of the figure, there are a $500 \mathrm{kV}$ pulse thermionic electron gun, a $238 \mathrm{MHz}$ subharmonic buncher, a $476 \mathrm{MHz}$ booster, a $1428 \mathrm{MHz}$ third-harmonic correction cavity, a $1428 \mathrm{MHz}$ alternating periodic structure rf cavity, and a $5712 \mathrm{MHz}$ fourth-harmonic correction cavity in the injector. Then, bunch compressor 1, four $2856 \mathrm{MHz}$ acceleration units, bunch compressor 2, $125712 \mathrm{MHz}$ acceleration units, bunch compressor 3, and $525712 \mathrm{MHz}$ acceleration units are laid. After these, three undulator beam lines continue. For example, beam line 3 has 21 undulators. At the end of SACLA, there is an experiment hall. 
TABLE I. Tolerance of the phase stability of the individual cavities.

\begin{tabular}{lccc}
\hline \hline & \multicolumn{1}{c}{$\delta V / V$} & $\delta \phi$ & $\delta t$ \\
Cavity & $\%$ in rms) & (degree in rms) & $(\mathrm{ps}$ in rms) \\
\hline 238 MHz SHB & \pm 0.01 & \pm 0.01 & \pm 0.12 \\
$476 \mathrm{MHz}$ booster & \pm 0.01 & \pm 0.02 & \pm 0.12 \\
$L$-band correction cavity & \pm 0.03 & \pm 0.06 & \pm 0.12 \\
L-band APS cavity & \pm 0.01 & \pm 0.06 & \pm 0.12 \\
$C$-band correction cavity & \pm 0.1 & \pm 0.06 & \pm 0.049 \\
$S$-band accelerator & \pm 0.01 & \pm 0.1 & \pm 0.097 \\
24 C-band accelerator & \pm 0.01 & \pm 0.2 & \pm 0.097 \\
$\quad$ (upstream) & & & \\
104 $C$-band accelerator & \pm 0.01 & \pm 0.5 & \pm 0.24 \\
$\quad$ (downstream) & & & \\
\hline \hline
\end{tabular}

technology of the SCSS test accelerator using the in-phase and quadrature (IQ) rf manipulation method. This $50 \mathrm{fs}$ short-term temporal jitter in the test accelerator almost reached the demanded temporal stabilities of the acceleration cavities for SACLA, given in Table I.

In order to guarantee the success of the SACLA construction, we should consider decreasing the short-term temporal jitter to a value below one-third of the achieved jitter value, at least. The temporal drift value was also insufficient to the requirements, when we consider the peakto-peak values (e.g., 6 sigma) given in Table II. Therefore, the effect from the environmental condition to the timing and LLRF system of SACLA should be reduced for adapting to the above-mentioned demands. Furthermore, the size difference between the SCSS test accelerator with about $50 \mathrm{~m}$ long and SACLA with about $700 \mathrm{~m}$ long was big. A reference $r f$ and timing signal transmission method and a LLRF system for SACLA should meet this size difference, such as by using low-loss signal transmission by optical fibers. For attaining the demands for SACLA, instrument developments in order to improve the temporal stability, like the rf phase, were necessary. Examples of the developed items are an ultralow-noise power supply used to drive the LLRF instruments, precise temperature control within $0.01 \mathrm{~K}$ for the timing and LLRF instrument enclosures, the use of thermal compensation rf devices, and an optical-fiber signal transmission system with opticallength control. These developments were conducted during SACLA's construction. In this paper, we describe the constructed timing and LLRF system for SACLA, the developed instruments, and its system performance, as one of the possible examples in order to realize the XFEL.

\section{TIMING AND LLRF SYSTEM}

\section{A. System configuration}

For realizing the stability demands, the timing and LLRF system for SACLA, as shown in Fig. 2, was developed and constructed. The configuration of the system is based on that of the SCSS test accelerator. The system mainly comprises master and end timing and LLRF terminal stations. There is a very low-noise master oscillator (MOSC) at the master terminal station in order to drive all of the instruments, which need reference continuous wave (cw) rf signals for their operation. The needed signals are 238, 476, 1428, 2856, and $5712 \mathrm{MHz} \mathrm{cw}$ rfs, and a $5712 \mathrm{MHz}$ cw rf with phase shift keying (PSK) [9] modulated by trigger pulses. Optical transmitters (OPT TXU-A and OPT TXU-C) having a role of electrical and optical (E/O) conversion are connected to the MOSC. The output signals of the optical transmitters are fed into phasestabilized optical fiber (PSOF) cables [10]. These cables have a low thermal optical-length coefficient of $2 \mathrm{ppm} / \mathrm{K}$. The signals in the optical fibers are transmitted by the wavelength division multiplex (WDM) method [11,12]. This WDM method merges the above-mentioned optical signals outputted from the MOSC into one optical fiber. These optical-fiber cables are installed into water-cooled and temperature-stabilized ducts and distributed to each of the end-terminal stations. These end-terminal stations are located near by individual high-power rf sources, such as pulse klystrons, and have water-cooled and temperaturestabilized 19-inch electric industry alliance (EIA) enclosures including instruments, such as LLRF components. Fiber-length control [13] compensates for the thermal expansions of a PSOF's optical length in order to mitigate any rf phase drift of the acceleration cavities. At the endterminal stations, an rf distributer (RF-DIS) having optical/ electrical $(\mathrm{O} / \mathrm{E})$ converters receives the optical signals outputted from OPT TXU-A and/or C. This RF-DIS converts the optical signals to electrical signals for driving the high-power rf source and instruments. One of the rf output from the RF-DIS is connected to an IQ modulator in order to manipulate an rf phase and amplitude and in order to make an $\mathrm{rf}$ pulse from the $\mathrm{CW}$ rf signal. The output signal of the IQ modulator is then fed into a $500 \mathrm{~W}$ solid-state amplifier, which drives the klystron. At some of the rf end-terminal stations in the injector, the IQ modulators for other frequency signals are connected to medium-power rf amplifiers in order to drive cavities, such as a $238 \mathrm{MHz}$ subharmonic buncher (SHB) cavity, a $476 \mathrm{MHz}$ booster cavity, and a $1428 \mathrm{MHz}$ correction cavity.

The original timing generator, which is called a master trigger unit (MTU), generates a pulse train with such a frequency of $60 \mathrm{~Hz}$. The output pulse train is used in order to activate accelerator instruments; it also decides the accelerator repetition cycle. The MTU, which is the module of a VERSAmodule Eurocard bus (VME), is placed near the MOSC, driven by the $238 \mathrm{MHz}$ rf signal for the SHB and synchronized to commercial ac power of $60 \mathrm{~Hz}$. The output pulses of the MTU are fed into OPT TXU-A and OPT TXU-C. The trigger pulse modulates one of the $5712 \mathrm{MHz}$ rf signals by the PSK method. After these 


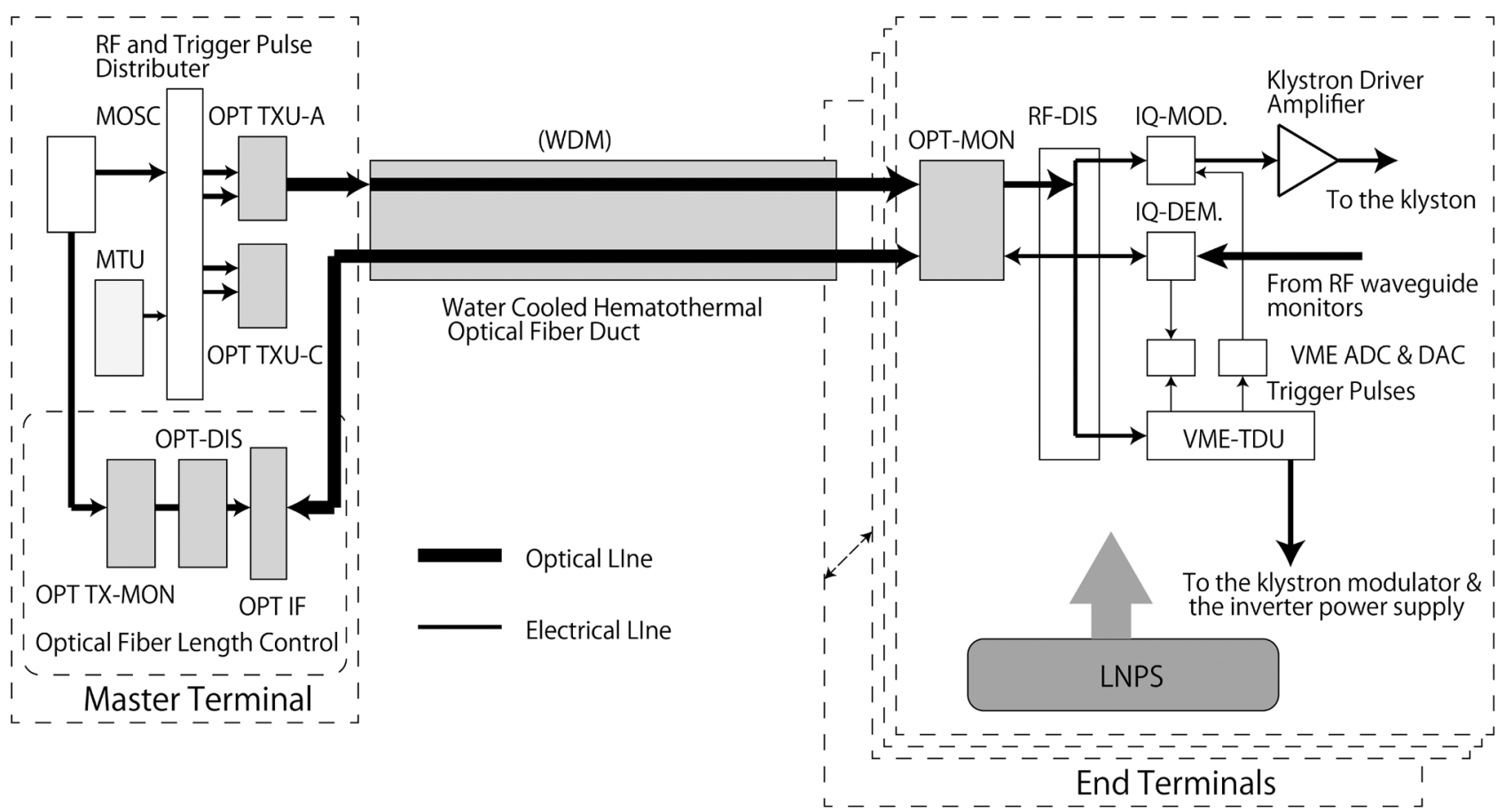

FIG. 2. Timing and LLRF system of SACLA. There are the MOSC, OPT TXU-A, and OPT TXU-C at the master terminal. The MOSC generates 238, 476, 1428 ( $L$-band), 2856 ( $S$-band), and $5712 \mathrm{MHz}(C$-band) rf signals. The MTU, OPT TXU-A, and OPT TXU-C output a $5712 \mathrm{MHz}$ rf signal with PSK-modulated trigger pulses. The PSOF cables transmit these signals by the WDM method. At the end-terminal stations, there is the RF-DIS. The rf output of the RF-DIS is fed into high-power rf sources through the IQ modulator. The trigger pulse is distributed to activate instruments, such as a klystron modulator and a data-acquisition (DAQ) system.

treatments, these pulses are transmitted to the end-terminal stations by the PSOFs, as mentioned above. At the endterminal station, the RF-DIS converts the PSK modulated optical rf signal to an electrical signal, and PSK demodulation is applied to pick up the trigger pulse from the phase (PSK) modulated $5712 \mathrm{MHz}$ rf signal. The trigger pulse is distributed to activate instruments, such as a klystron modulator and a data-acquisition (DAQ) system.

Ultralow noise and stable power supplies (LNPS) provide the dc powers for the above-mentioned timing and LLRF instruments. This LNPS allows for very small amplitude and phase noise of an rf field in the cavity and was specially developed in order to obtain stable laser beams driven by stable electron beams.

\section{B. Master-terminal station}

The master-terminal station equips the MOSC, OPT TXU-A, OPT TXU-C, and MTU. OPT TXU-A and OPT TXU-C were newly designed for SACLA. The other instruments are basically the same as those of the SCSS test accelerator [7]. Hence, in this section, we explain only the newly designed parts mentioned below, except for the established instruments.

\section{1. $O P T T X U-A$ and $O P T T X U-C$}

In the design stage of the optical rf reference-signal transmission, there were two candidates of optical transmission methods. One was a laser modulated by a reference cw rf signal; the other was an optical comb signal modulated by a cw rf signal. Since the $\mathrm{cw}$ rf signal is necessary in order to drive the klystron, a small $\mathrm{cw}$ portion (at a specific frequency) of all the power should be picked up from the optical comb signal with a bandpass filter. On the other hand, the $\mathrm{CW}$ optical signal includes a rich powerspectrum intensity of the $\mathrm{cw}$ rf necessary for the klystron. Hence, the noise intensity after picking up the needed cw rf signal from the cw optical signal is much smaller than that of the optical comb signal. In other words, this means that we can get the $\mathrm{cw}$ rf signal with a good signal-to-noise ratio. For this advantage of low noise, we chose optical cw rf transmission for the timing and LLRF system of SACLA. Reference [14] minutely discusses this argument for selecting the optical transmission method.

After this argument, the optical transmitter of TXU-A using the $\mathrm{cw}$ rfs, as shown in Fig. 3, was designed and built. At first, we used a Mach-Zehnder interferometer $\left(\mathrm{LiNbO}_{3}\right.$, lithium niobate, LN modulator) $[15,16]$, as an optical rf modulator for TXU-A. However, the thermal amplitude drift of this interferometer, connecting to a lasing intensity change, was not ignorable in SACLA's practical operation. These rf phase and amplitude drift values are $-2.18^{\circ} / \mathrm{K}$ and $-0.66 \% / \mathrm{K}$ at $5712 \mathrm{MHz}$, respectively. These values were measured at the output of a $1 \mathrm{~W}$ erbium doped fiber optical amplifier (EDFA) [17,18] to be connected after the LN 


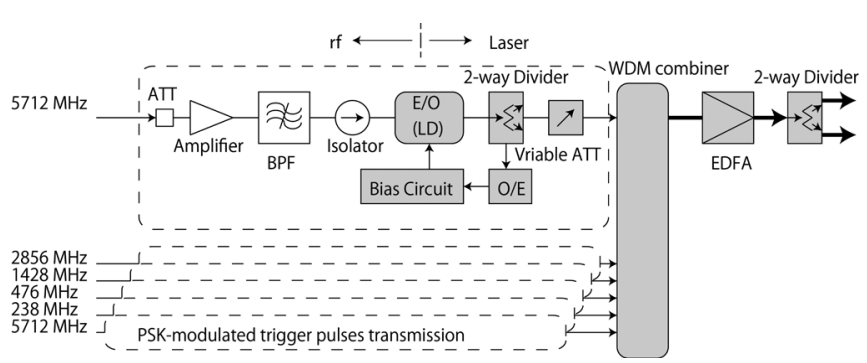

FIG. 3. Configuration of OPT TXU-A. The main function of OPT TXU-A is to convert electrical signals to optical signals. OPT TXU-A equips the laser diodes (LD) directly modulated by rf signals, such as the $5712 \mathrm{MHz}$ signal and the WDM combiners to merge multioptical rf signals. The bias voltage for the laser diode is controlled so as to stabilize its optical power level.

modulator. Therefore, the configuration of the E/O converter was changed so as to use a distributed feedback laser diode (DFB-LD) directly modulated by the $\mathrm{cw}$ rf signal, such as a frequency of $5712 \mathrm{MHz}$. This direct rf modulation method applied to the DFB-LD has thermal rf phase and amplitude drift values of $-3.5^{\circ} / \mathrm{K}$ and $-0.075 \% / \mathrm{K}$ at $5712 \mathrm{MHz}$, respectively. These values were also measured at the EDFA output, and the amplitude variation was almost 10 times lower than that of the LN modulator. The configuration of TXU-A finally became as follows, by the above-mentioned development process. This transmitter comprises a DFB-LD, an EDFA, optical dividers, and a WDM optical signal combiner. The configuration of TXU$\mathrm{C}$ is almost the same as that of TXU-A. These components of the optical transmitter are installed into the unit of a 19inch EIA enclosure. The specifications of the optical transmitters are tabulated in Table II.

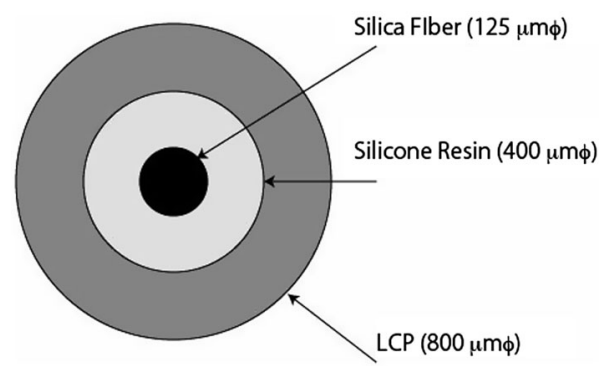

FIG. 4. Cross-sectional view of a phase-stabilized optical fiber (PSOF) cable. A liquid crystal polymer (LCP) that compensates the positive thermal coefficient of the silica index of refraction is coated around the outer surface of the resin.

\section{Optical-fiber signal transmission line}

In order to stably transmit the timing and rf signals, as previously mentioned, we use the PSOF. A cross-sectional view of the PSOF is shown in Fig. 4 [10]. Liquid-crystal polymer (LCP) in order to compensate the thermal optical length change of a quartz optical fiber is coated on the outer surface of the quartz fiber. The PSOF has a positive thermal optical-length coefficient of $2 \mathrm{ppm} / \mathrm{K}$. These optical-fiber cables are installed in water-cooled hemathermal ducts, as shown in Fig. 5 [11], in order to mitigate any environmental temperature change around them. These ducts have structures with dual metal surfaces in order to make a thermal equipotential and dual insulation layers using glass wool surrounding them. The function of these structures is to make further thermal isolation and a delayed response time to any outside temperature change compared with ordinal one-layer thermal isolation using glass wool. Most of the optical cables are installed in the duct depicted on the left

TABLE II. Specifications of the optical transmitter, TXU-C. The phase and amplitude jitter and drift specifications at the $238 \mathrm{MHz}$ signal are below those for the $5712 \mathrm{MHz}$ case.

\begin{tabular}{lc}
\hline \hline rf frequency & 5712 and $238 \mathrm{MHz}$ \\
\hline $\begin{array}{l}\text { Input rf power level } \\
\text { Output optical power level from } 1 \text { to } 8\end{array}$ & $0 \mathrm{dBm} \pm 0.01$ \\
$\quad$ (three optical waves are merged by the WDM) & $18.8 \mathrm{dBm}$ each \\
Output optical power level from 9 to 16 & $21.8 \mathrm{dBm}$ each \\
$\quad$ (three optical waves are merged by the WDM) & \\
Transmit optical wavelength 1 & \\
Transmit optical wavelength 2 & $1554.13 \mathrm{~nm}$ for the $5712 \mathrm{MHz} \mathrm{rf}$ \\
Transmit optical wavelength 3 & $1547.72 \mathrm{~nm}$ for the $238 \mathrm{MHz} \mathrm{rf}$ \\
& $1542.94 \mathrm{~nm}$ for the PSK modulated \\
Phase jitter at the $5712 \mathrm{MHz} \mathrm{rf}$ & $5712 \mathrm{MHz} \mathrm{rf}$ for the trigger pulses \\
Amplitude jitter at the $5712 \mathrm{MHz} \mathrm{rf}$ signal & Below $0.5^{\circ}$ in rms \\
Long-term phase drift at the $5712 \mathrm{MHz} \mathrm{rf}$ & Below $0.1 \%$ in rms \\
Long-term amplitude drift at the $5712 \mathrm{MHz}$ rf & Below $0.5^{\circ}$ \\
Thermal phase drift at the $5712 \mathrm{MHz} \mathrm{rf}$ & Below $0.1 \%$ \\
Thermal amplitude drift at the $5712 \mathrm{MHz}$ rf & $1^{\circ} / \mathrm{K}$ \\
Input rf impedance & $0.5 \% / \mathrm{K}$ \\
Optical signal distribution number & $50 \Omega$ \\
\hline \hline
\end{tabular}




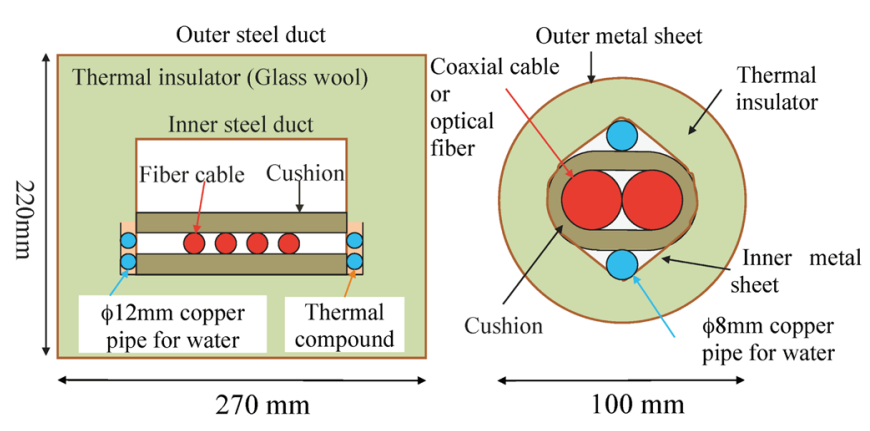

FIG. 5. Cross-sectional views of the water-cooled hemathermal optical-fiber ducts. The ducts have dual metal surfaces to make a thermal equipotential on them and dual insulation layers using glass wool around the optical-fiber cables. The function of these structures is to make better thermal isolation and a delayed response time to an outside temperature change than those of ordinal one-layer thermal isolation using glass wool.

side of Fig. 5. The other, where installation space is limited, is installed into the duct shown on the right side of Fig. 5.

The temperature-regulation performance of the duct depicted on the left side of Fig. 5 is shown in Fig. 6. The duct is cooled by water within a temperature change of $\pm 0.1 \mathrm{~K}$. The regulated temperature change in the duct is less than $\pm 0.1 \mathrm{~K}$. This temperature regulation performance conducts a PSOF length change of less than $0.1 \mathrm{~mm}$ for a length of $1 \mathrm{~km}$.

\section{Optical-fiber-length control}

Control system.-In order to compensate the thermal optical-length expansion of the PSOF, an optical-length control system using the Michelson interferometry method [19] was employed. Figure 7 describes the block diagram of a fiber-length control system. This system comprises a coarse length control subsystem with a wide dynamic range of $3 \mathrm{~mm}$ and a fine length control subsystem with a narrow dynamic range of $750 \mathrm{~nm}$. The coarse length control is

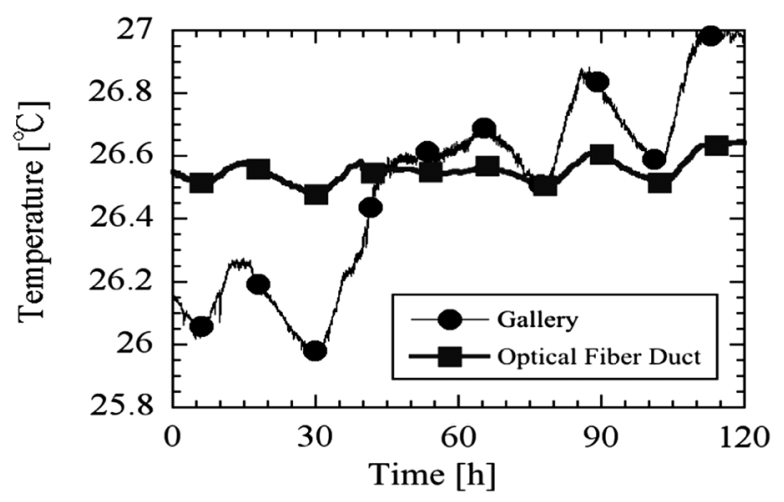

FIG. 6. Temperature stability of the water cooled hemathermal duct on the left side of Fig. 5 is depict. The temperature change of the ducts is within $\pm 0.1 \mathrm{~K}$, while the temperature outside of the duct (klystron gallery) changes by more than $1 \mathrm{~K}$. based on the wavelength of a $45 \mathrm{GHz}$ rf signal, which is the difference between two optical wavelength signals of 1552.52 and $1552.89 \mathrm{~nm}$. The fine length control is based on an optical wavelength of $1548.96 \mathrm{~nm}$. A frequencystabilized laser generates this reference laser light for the fine control. The optical-length measurement procedure of the PSOF is as follows. The reference laser light is injected into the PSOF in order to measure its optical length and is transmitted along the PSOF. The light is reflected back from the optical-fiber end by a Faraday rotation mirror (FRM) to the beginning point, where the reference light is injected. The FRM is installed in an optical monitor unit (OPT-MON), whose details will be described in a later section. At the beginning point, an optical polarized beam splitter (PBS) separates a part of the reflected light, and an optical divider (OPDIV) also splits a part of the injected reference forward light. An acoustic optical modulator (AOM) modulates the part of the injected light by a $238 \mathrm{MHz}$ rf signal. PIN photodiodes (O/E) convert both the forward and reflected laser beams into electrical signals. A digital phase detector used to measure the optical length of the PSOF compares the phase relation between the signals electrically converted from the forward and reflected light beams. The phase-detected signal is fed into an optical-fiber stretcher [20] in order to stabilize the PSOF's optical length through a feedback control filter. This filter is based on the proportional, integrate and differential (PID) feedback-control method. Figure 8 shows the performance of our optical-fiber-length control system. Our developed optical-fiber-length control system (fine control) reduces the optical-length change of the PSOF perturbed due to the environmental conditions, such as the temperature, humidity, and vibration, up to below $0.5 \mu \mathrm{m}$ for $1.5 \mathrm{~km}$ in total length. In the following sections, we describe the details of the individual components for the optical-fiber-length control.

Optical transmitter.-An optical transmitter (OPT TXMON) that includes a frequency reference laser source for the optical-fiber-length control is installed in the masterterminal station. The configuration of OPT TX-MON is shown in Fig. 9. An external cavity laser diode (ECLD) used for fine fiber-length control emits a $1548.96 \mathrm{~nm}$ reference laser beam, whose frequency is locked to the absorption line of hydrogen cyanide $\left(\mathrm{H}^{13} \mathrm{C}^{14} \mathrm{~N}\right)$ gas for frequency stabilization. The laser source for the coarse fiber-length control is also an ECLD operated at $1552.52 \mathrm{~nm}$. Three stages of a 2 times rf multiplier generate an rf signal at $45 \mathrm{GHz}$ from the $5712 \mathrm{MHz}$ signal in order to measure the coarse optical-fiber length. This $45 \mathrm{GHz}$ signal is fed into a Mach-Zehnder interferometer in order to produce two sideband light beams from the incident $1552.52 \mathrm{~nm}$ laser light. These sideband wavelengths are 1552.15 and $1552.89 \mathrm{~nm}$, which have a difference frequency of $91.4 \mathrm{GHz}$, respectively; $45.7 \mathrm{GHz}$, which is half 


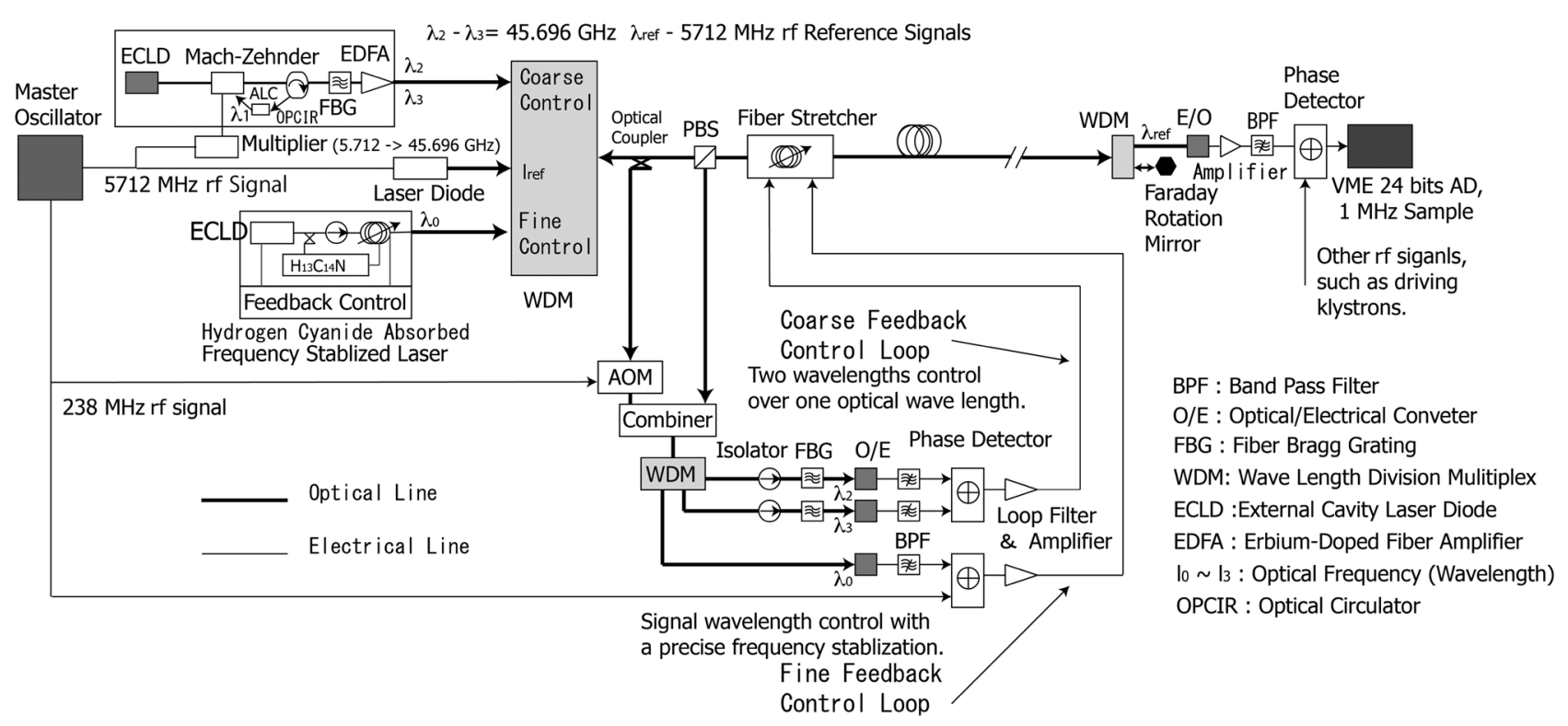

FIG. 7. Block diagram of the optical-fiber-length control system. The system comprises a coarse length control subsystem and a fine length control subsystem.

of $91.4 \mathrm{GHz}$, is the frequency difference between the 1552.52 and $1552.89 \mathrm{~nm}$ laser beams. The WDM method merges the output light beams of the frequency-stabilized laser source for fine length control and the laser source for coarse length control. These merged optical signals are inputted to EDFA and amplified for adapting long-line signal transmission, such as more than $1 \mathrm{~km}$.

Optical interferometry unit.-An optical interferometry unit (OPT-IF) using the Michelson interferometry method allows one to measure the fiber-optical-length change affected due to the environmental conditions, such as the temperature change. Two-way OPDIVs, a PBS, an AOM, a WDM combiner, a WDM divider, phase detectors, PID feedback-control filter circuits, optical-fiber stretchers, and high-voltage drivers for the stretchers mainly configure the

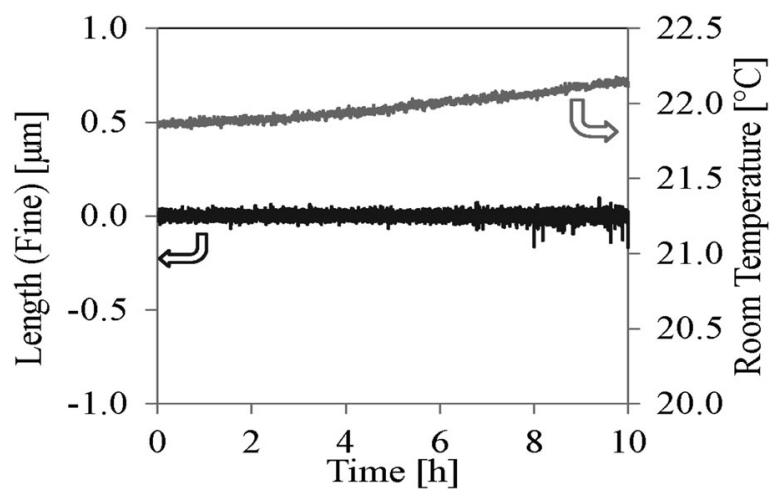

FIG. 8. Performance of the fine fiber-length feedback control. The fiber length is controlled within $0.3 \mathrm{fs}(<0.1 \mu \mathrm{m})$ for $10 \mathrm{~h}$, even though the environmental temperature around the fiberlength control system changes by about $0.5 \mathrm{~K}$.
OPT-IF, as shown in Fig. 10. The OPDIV has a role of distributing the incident forward light to the optical fiber and to the phase detector for the fiber-length measurement. The PBS has a role of splitting the light reflected from the FRM at the end terminal for phase detection. When the phase reflecting the optical-fiber-length change between the forward and backward light beams is detected by the heterodyne method, the incident laser light should be modulated by the $\mathrm{cw}$ rf signal. We choose the $238 \mathrm{MHz}$ signal for the SHB as this rf signal, because of the temporal fundamental frequency of the SACLA accelerator. The AOM performs this modulation. The reflected light is fed into a digital phase detector, which basically comprises exclusive OR (XOR) logic gates and an integration circuit. The optical-fiber stretchers and high-voltage drivers for the stretchers are actuators used to regulate the fiber length.

\section{End-terminal station for the high-power $\mathrm{rf}$ source}

\section{System configuration of the end-terminal station}

Figure 11 shows an example of instrument arrangements in a 19-inch electric industry alliance (EIA) enclosure at the end-terminal stations for the timing and LLRF system. This enclosure is a water-cooled and temperature-stabilized rack.

Figure 12 also depicts an instrument block diagram of the enclosure settled around a $C$-band high-power klystron. The rf and timing signals fed into the end terminal, such as the $5712 \mathrm{MHz}$ signal, the $238 \mathrm{MHz}$ signal, and the PSKmodulated timing pulse, are transmitted from the previously mentioned master terminal. These signals are split at the end terminal, into individual accelerator instruments, such as the high-power rf source and the DAQ system. 


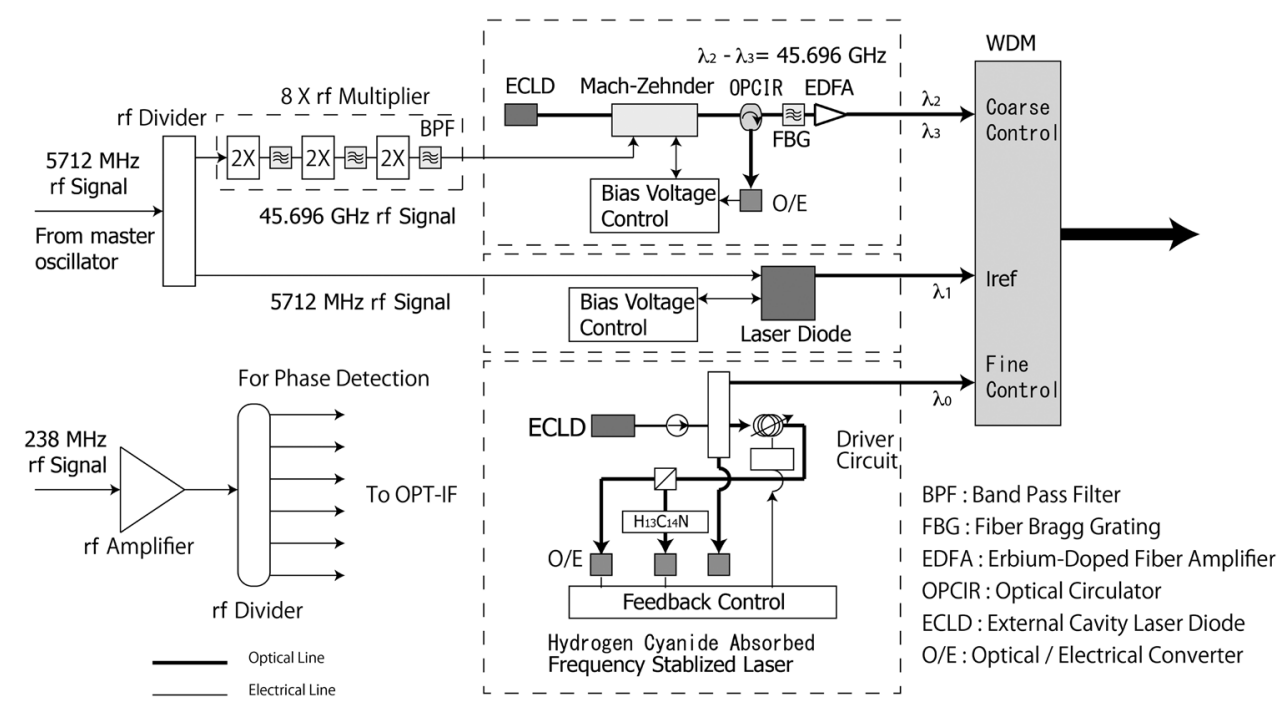

FIG. 9. Configuration of OPT TX-MON. This optical transmitter has three laser sources for the fine and coarse feedback control loops and $5712 \mathrm{MHz}$ reference off transmission. One laser source is to transmit the $45.7 \mathrm{GHz}$ rf signal for the coarse control. The Mach-Zehnder module modulates light from the ECLD by the $45.7 \mathrm{GHz}$ rf signal. The other is to transmit an optical-wavelength reference signal for fine control. A laser generated by the other ECLD is locked to the absorption line of the $\mathrm{H}^{13} \mathrm{C}^{14} \mathrm{~N}$ gas cell for the fine control. The third one is a further ECLD directly driven by a $5712 \mathrm{MHz}$ reference rf signal.

An RF-DIS, an OPT-MON, a DAQ system cage, an IQ demodulator, an IQ modulator, a $C$-band $500 \mathrm{~W}$ klystron driver amplifier, and a LNPS are included in the 19-inch enclosure for the end terminal. The VME modules, such as a 16-bit $238 \mathrm{MHz}$ rf driven ADC, a trigger delay unit (TDU), an interrupt register, and a CPU, are installed in the DAQ system cage. The RF-DIS receives the optical rf signals from the master-terminal station and converts from the optical signals to electrical rf signals. One of the rf signals outputted from the RF-DIS, such as a $5712 \mathrm{MHz}$ signal, is delivered to the IQ modulator for manipulating its rf phase and amplitude. A 16-bit $238 \mathrm{MHz}$ DAC module

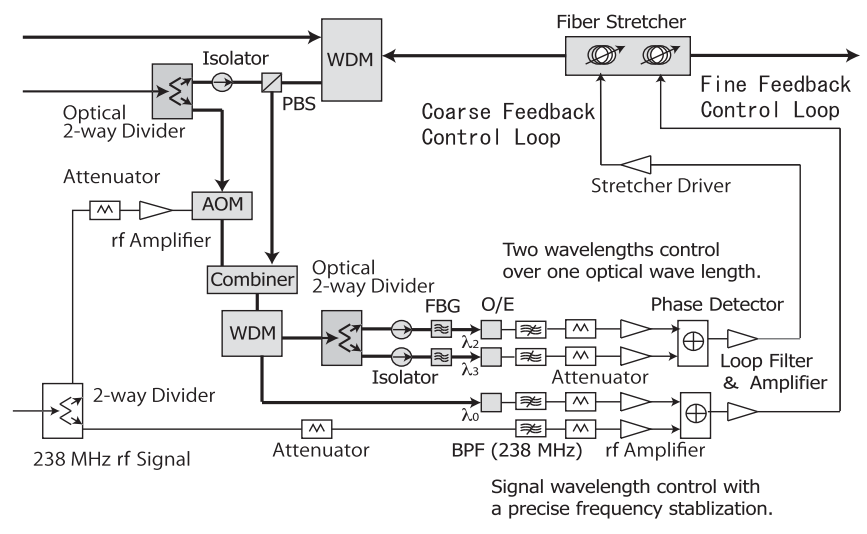

FIG. 10. Configuration of OPT-IF using the Michelson interferometry method. This system has both fine and coarse control. The coarse length control is based on the wavelength of a 45.7 GHz rf signal, which is the difference between two optical wavelength signals of 1552.52 and $1552.89 \mathrm{~nm}$. The fine length control is based on an optical wavelength of $1548.96 \mathrm{~nm}$. generates baseband signals, allowing manipulation for the IQ modulator. Then, the klystron driver amplifier amplifies the output rf power of the modulator from $1 \mathrm{~mW}$ to $500 \mathrm{~W}$ in order to drive the $C$-band klystron. On the other hand, other rf signals of 5712 and $238 \mathrm{MHz}$ are also fed into

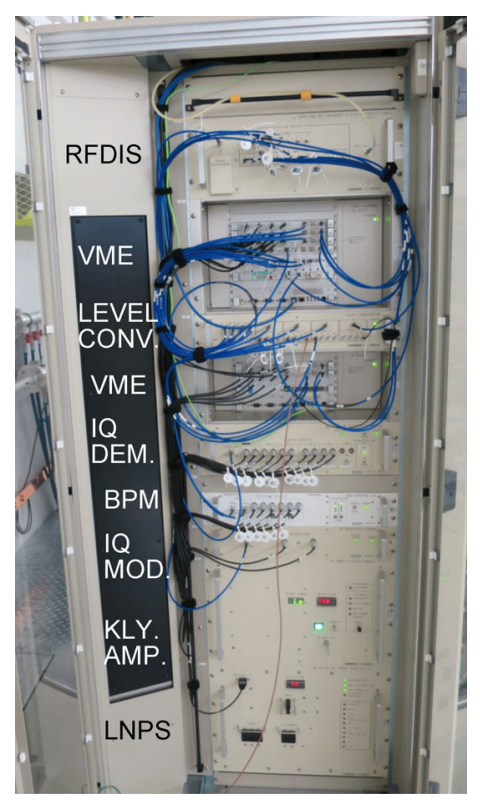

FIG. 11. Example of the instrument arrangements in the 19inch EIA enclosure at the end-terminal stations for the timing and LLRF system around the $C$-band high-power klystron. This enclosure is a water-cooled hematothermal rack. The RF-DIS, the VME crate, the level converter, the IQ demodulator, the BPM detection module, the IQ modulator, the klystron driving amplifier, and the LNPS are installed in the enclosure. 


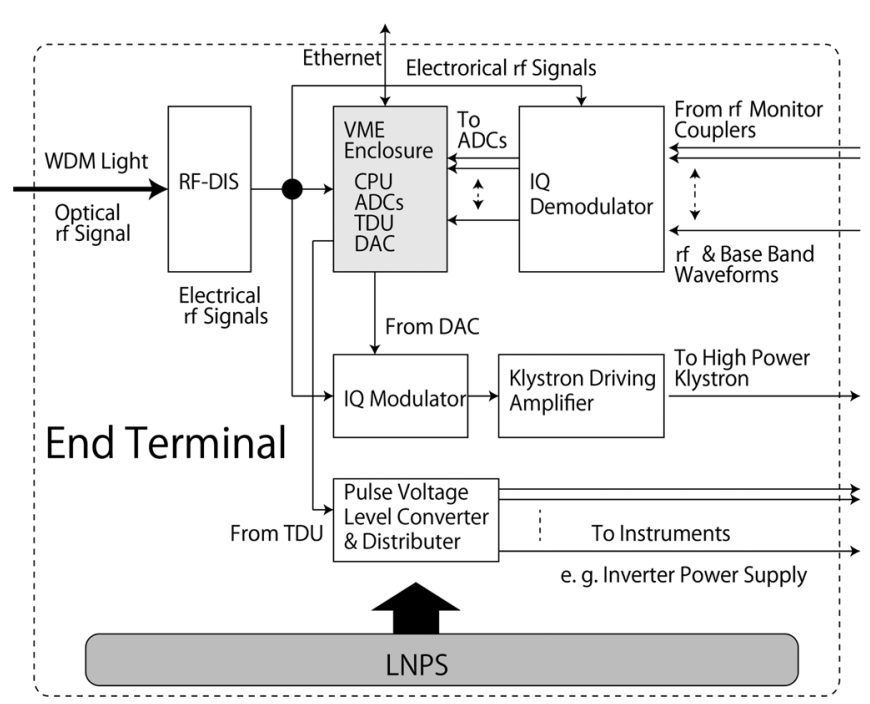

FIG. 12. Block diagram of instruments in the end-terminal station. The rf and timing WDM signals fed into the end terminal, such as the $5712 \mathrm{MHz}$ signal, the $238 \mathrm{MHz}$ signal and the PSKmodulated timing pulse, are transmitted from the master terminal. These signals are split into individual accelerator instruments, such as the high-power rf source and the DAQ systems.

16-bit ADCs for synchronously detecting the electron acceleration $\mathrm{rf}$ at the electron beam timing and the TDU in order to determine the activation timing of accelerator instruments. The output trigger pulse of the RF-DIS, which is demodulated from the PSK-modulated signal, is fed in to the TDU in order to deliver the pulses to many instruments. These trigger instruments are such as 16-bit ADCs, a klystron driver amplifier, and a high-voltage inverter power supply for a klystron modulator in the 19-inch enclosure. The trigger pulses synchronously activate these instruments in accordance with an accelerator repetition cycle.

The following sections describe the details of the timing and LLRF instruments in the end-terminal station. Detail explanations of similar instruments [7], to be used in the SCSS test accelerator, are omitted, since the instruments have almost the same feature between both cases of the SCSS test accelerator and SACLA.

\section{2. rf distributer (RF-DIS)}

Figure 13 depicts an internal block diagram of the RFDIS, which comprises optical and electrical (O/E) converters using PIN photodiodes, rf amplifiers, $r$ dividers, and a PSK demodulator using a phase detector. The RF-DIS has functions in order to receive the rf modulated optical signals, to convert from the optical signals to the electrical signals, and to distribute the converted electrical signals to the instruments contained in the enclosure. The PSK demodulator in the RF-DIS demodulates the PSK modulated optical rf signal of the master station to electrical trigger pules for the instruments. Figure 14 shows the single sideband phase-noise power spectrum of the $5712 \mathrm{MHz}$ rf

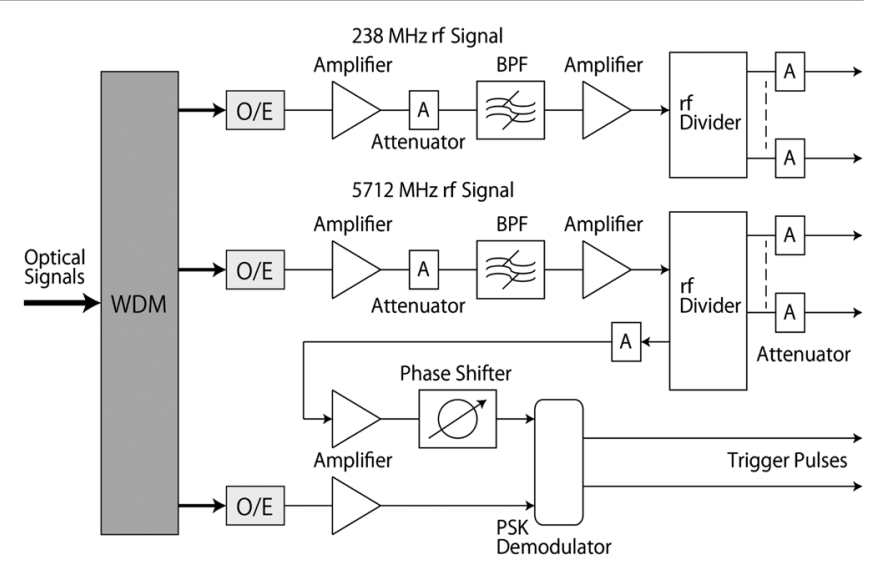

WDM: Wavelength Dlvision Multiplex O/E: Optical \& Electrical Converter BPF: Bandpass Filter

FIG. 13. Internal block diagram of the RF-DIS. The RF-DIS is composed of optical-to-electrical (O/E) converters, rf amplifiers, rf dividers, and a PSK demodulator.

signal at the electrical output of the RF-DIS. This noise spectrum was measured with the signal source analyzer (E5052, Agilent Technologies) [21]. The noise level of the RF-DIS is almost the same as that of the MOSC.

\section{Optical monitor unit (OPT-MON)}

The main function of the OPT-MON, as shown in Fig. 15, is the optical receiver for the optical-fiber-length control. This unit comprises a FRM, a fiber stretcher, and a high-voltage amplifier in order to drive the stretcher, as well as an rf divider and a $5712 \mathrm{MHz}$ phase frequency discriminator (PFD). The FRM reflects back the optical signal to the OPT-IF for the fiber-length control and rotates the optical polarization by $90^{\circ}$ in order to distinguish the reflected light from the other scattered light. In other word, the FRM compensates any linear-birefringence effects in the PSOF originating from round-trip light on the fiber in

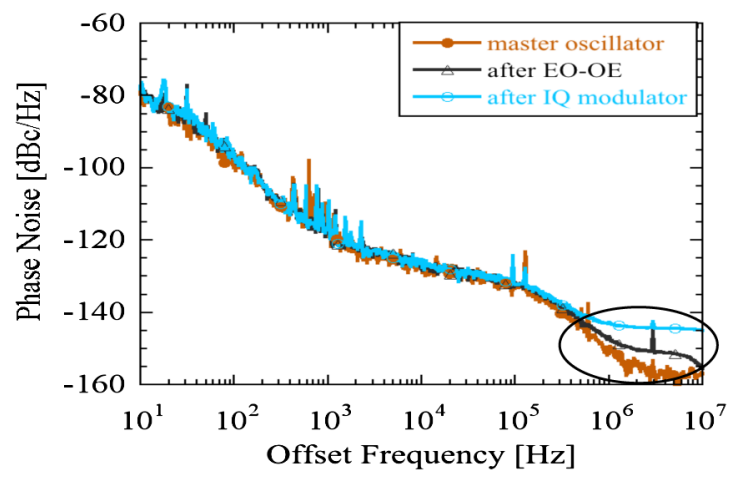

FIG. 14. Single side-band phase noise power spectrum of the $5712 \mathrm{MHz}$ rf signal at the electrical output of the RF-DIS. The noise level of the RF-DIS is almost the same as that of the MOSC. The noise-increasing part displayed in the circle between the MOSC and the IQ modulator corresponds to a temporal jitter of 7 fs. The RF-DIS and the IQ demodulator cause this noise increase measured by using a $\mathrm{cw}$ rf signal. 


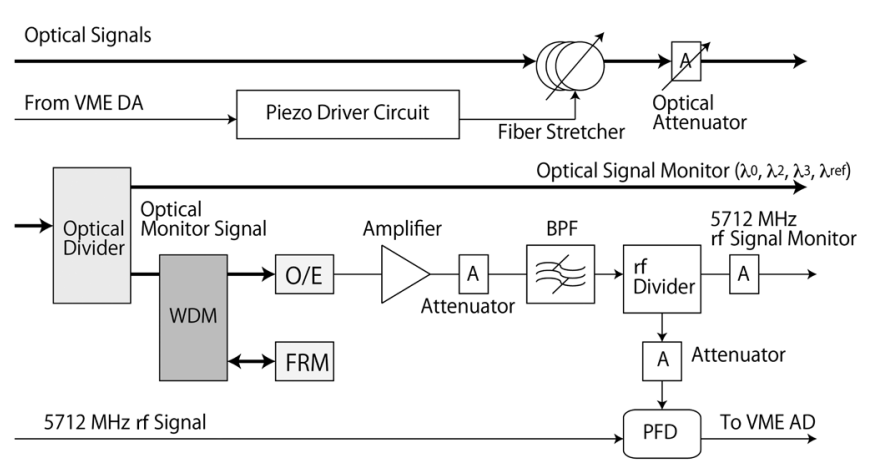

WDM: Wavelength Dlvision Multiplex O/E: Optical \& Electrical Converter BPF: Bandpass Filter FRM: Faraday Rotation Mirror PFD: Phase Frequency Discriminator

FIG. 15. Block diagram of OPT-MON. The main function of OPT-MON is the optical receiver for the optical-fiber-length control. This unit comprises a Faraday rotation mirror, a fiber stretcher, a high-voltage amplifier (piezo driver) to drive the stretcher, an rf divider, and a $5712 \mathrm{MHz}$ phase detector (phase frequency discriminator, PFD).

order to maintain a certain power (signal level) and polarization at the PBS [22]. The fiber stretcher is used to control the phases of the acceleration rf signals fed into the RF-DIS. The PFD is used for comparing the rf phase between a reference $5712 \mathrm{MHz}$ signal transmitted through the optical-length controlled fiber from the master station and a $5712 \mathrm{MHz}$ rf signal from the RF-DIS for the electron acceleration. This phase comparison allows mitigation of the phase drifts of all the acceleration rf signals at the end terminal.

\section{IQ modulator and demodulator}

The basic concept of the IQ modulator and the IQ demodulator [7] was considered when the SCSS test accelerator was constructed. The signal frequencies for IQ rf detections were 2856 and $5712 \mathrm{MHz}$. However, an IQ manipulation frequency for the instruments was originally $476 \mathrm{MHz}$. Hence, the IQ instruments for the test accelerator used the frequency down-conversion method. At the time, we could not employ a higher detection frequency than $476 \mathrm{MHz}$ because of a technical limitation of the mixer devices. The disadvantage of this down-conversion method was an increase in the number of necessary local reference signals in order to detect the rf signals by the heterodyne method [23]. In this case, a LLRF system should deliver at least two frequency signals for the detection. Therefore, in the case of SACLA, IQ modulation and demodulation using a single-frequency local signal are employed to reduce its distribution number.

Figure 16 shows an example of the configuration of the IQ demodulator. An IQ demodulation IC in the figure is a HMC218 MSB made by Hittite Microwave Corporation [24]. This single-frequency manipulation is one of the main features of these IQ modulator and demodulator devices. The other feature of these instruments is to reduce the

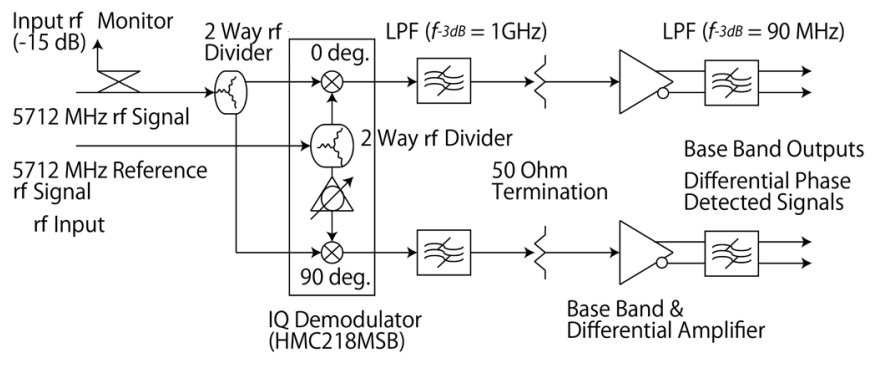

FIG. 16. Configuration of the IQ demodulator. This uses an IQ demodulation IC of HMC218 MSB made by Hittite Microwave Corporation.

cross-correlation effect between the rf phase and amplitude control. This reduction is achieved by a numerical calculation in order to secure the orthogonality between the I and $Q$ values taken with the instruments. An rf network analyzer measured the rf phase and amplitude cross term, and its cross term was compensated by a calibration calculation using the measured data [25]. Figure 17 shows the phase and amplitude detection errors of the IQ demodulator before and after the cross-term reduction calibration, as a function of ideal phase detection values. After the calibration, the output rf phase and amplitude variations were reduced from $5^{\circ}$ and $5 \%$ to within $1^{\circ}$ and $1 \%$ in accordance with $360^{\circ}$ controlled phase changes, respectively. Figure 14 shows the output noise level at the IQ modulator output, which includes noises caused by the MOSC origin and the optical-fiber signal transmission

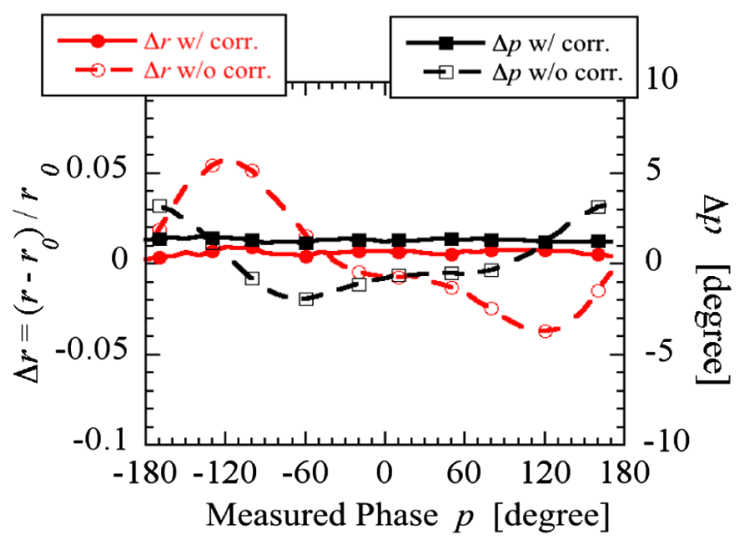

FIG. 17. Radio frequency phase and amplitude detection errors of the IQ demodulator before and after a cross-term reduction calibration between the phase and amplitude, as a function of the ideal phase detection values. $P$ is the phase, $\Delta p$ is the phase error, $r$ is the amplitude, and $\Delta r$ is the amplitude error. The dashed line with open circles shows the amplitude without any correction; the dashed line with open squares shows the phase without any correction. The solid line with solid circles depicts the amplitude with a correction, and the solid line with a solid square depicts the phase with a correction. After a cross-term reduction calibration, the output rf phase and the amplitude variations are reduced from $5^{\circ}$ and $5 \%$ to within $1^{\circ}$ and $1 \%$ in accordance with $360^{\circ}$ controlled phase changes, respectively. 


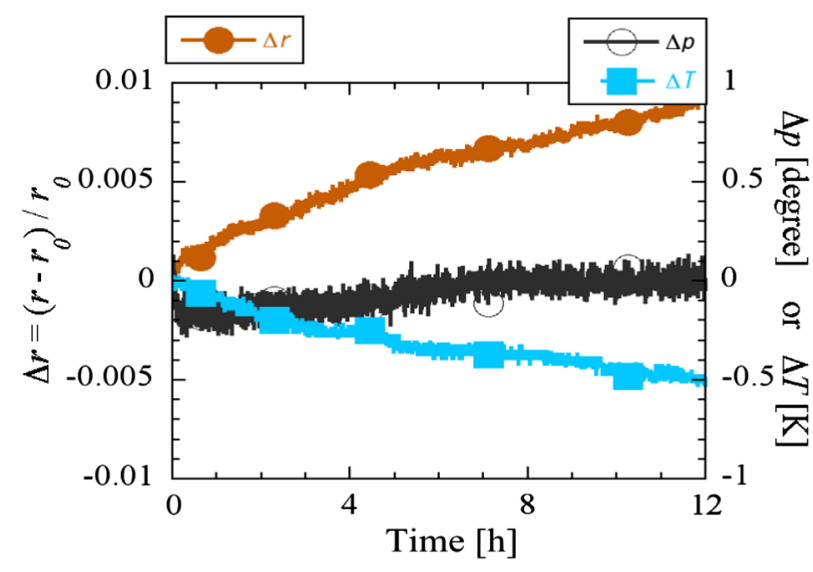

FIG. 18. Output amplitude (upper line) and phase (middle line) drifts of the IQ control system and an environmental temperature change (bottom line) around the demodulator, as a function of time. $\Delta p$ is the phase drift, $\Delta T$ is the temperature drift, $r$ is the amplitude, and $\Delta r$ is the amplitude drift.

system. Figure 18 describes the output phase and amplitude drifts of the IQ rf control system comprising equipment sequentially connected among the DAC module, the IQ modulator, the IQ demodulator, and the ADC module. These drift data were taken with a VME computer through the ADC module. The phase drift is about $0.4^{\circ} / \mathrm{K}$ and the amplitude drift is about $2 \% / \mathrm{K}$. The output noise level and drift of the IQ modulator are almost the same levels of those of the IQ demodulator. Each drift of the modulator and demodulator could be less than half of the previously mentioned values.

\section{16-bit, $238 \mathrm{MHz} V \mathrm{VE} A D C$ and DAC modules for $r$ drive}

The VME-ADC module [7,26], as shown in Fig. 19, was developed to digitize the signals including the rf phase and amplitude information detected with the IQ demodulator. A VME-DAC module [7] was also developed to manipulate the rf phase, amplitude, and pulse modulation with the IQ modulator. The designs of both VME modules were based on the concepts of those of the SCSS test accelerator. These individually have four input or four output channels, a 16bit dynamic range, a function of wave form detection or generation synchronized by a $238 \mathrm{MHz}$ clock, and a $30 \mathrm{MHz}$ frequency bandwidth at their analog inputs or outputs. The input and output ports of the ADC and DAC have differential lines with a characteristic impedance of $100 \Omega$ so as to reduce any external common-mode noise. One channel of the ADC module has two analog-to-digital (AD) converter integration circuits (IC, product of linear technology, LTC2208) driven by a clock of up to $119 \mathrm{MHz}$, each. The two ADC ICs alternatively work to realize $238 \mathrm{MHz}$ data acquisition. It also has a field-programmable gate array (FPGA) IC to control data taking by the ADC ICs and a VME bus. The DAC module almost has the same

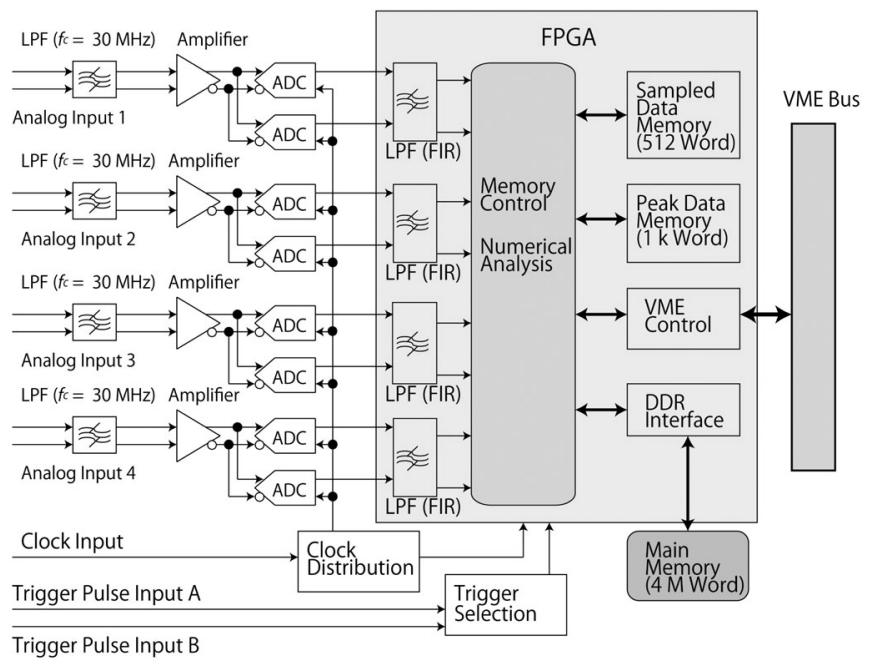

FIG. 19. Internal block diagram of the VME 16-bit ADC module. This module is used to detect the rf phase and amplitude. The ADC has four input channels, a 16-bit dynamic range, a function involving wave-data taking synchronized by a $238 \mathrm{MHz}$ clock, and a $30 \mathrm{MHz}$ frequency bandwidth at their analog inputs. The input ports of the ADC are differential lines with a characteristic impedance of $100 \Omega$.

hardware configuration as that of the ADC module and comprises one digital-to-analog (DA) converter IC for each channel and a FPGA IC for obtaining the almost-same control functions as that of the ADC. The DAC (product of analog devices, AD9779) works to realize $238 \mathrm{MHz}$ synchronized data output.

The ADC module is like an oscilloscope without a display to digitize an arbitrary wave form. Its digitization for the input wave form starts when an external trigger pulse is fed into the module. This external trigger pulse is synchronized to an accelerated electron beam and outputted from the TDU. The VME-ADC module can detect and store an error wave form out of tolerance referred to a reference wave form that is memorized in advance. The tolerance value, such as $10 \%$, is also defined in advance. The DAC module has functions that make a pulse having an arbitrary wave form, such as $2 \mu \mathrm{s}$ in width. These functions are for driving the IQ modulator and for providing PSK operation during an rf-pulse flattop for rf pulse compression with SLED (SLAC energy doubler) [27]. One set of the two output channels of the DAC module can handle the I and Q output voltages reflecting on the rf phase and amplitude information for controlling the IQ modulator, respectively. This DAC module every time generates an arbitrary shape pulse triggered by the external pulse.

We finally obtained a dynamic range devoid of noise in the ADC and DAC modules; the range is about 12.5 bits $\left(2 \times 10^{-4} \mathrm{~V}\right.$ resolution). The noise floor level on a frequency spectrum of the ADC output data, as shown in Fig. 20, is below $-90 \mathrm{dBV}$ in a region from $1 \mathrm{~Hz}$ to $119 \mathrm{MHz}$, and the DAC output almost has the same noise 


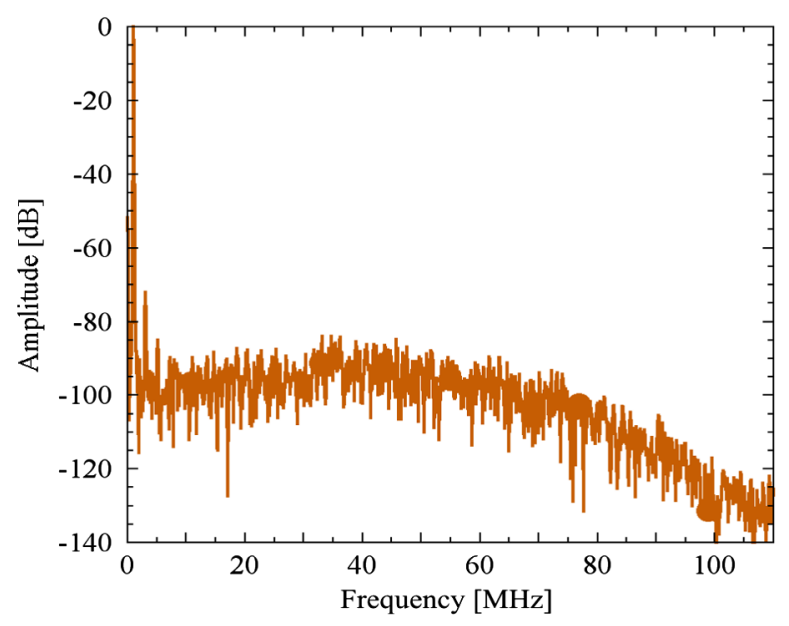

FIG. 20. Noise floor level of the frequency spectrum of ADC output data. In these data, a $1 \mathrm{MHz}$ sinusoidal signal with fullscale amplitude $( \pm 1 \mathrm{~V})$ is applied to the ADC input. The number of data points is 8192 . The noise floor level is below $-90 \mathrm{dBV}$ in the region from $1 \mathrm{~Hz}$ to $120 \mathrm{MHz}$.

floor level. These data with 8192 points (Fig. 20) were taken when a $1 \mathrm{MHz}$ sinusoidal signal with full-scale amplitude $( \pm 1 \mathrm{~V})$ was added to the input port. The thermal output voltage drift of the DAC and the voltage conversion drift of the ADC are less than $2 \times 10^{-4} \mathrm{~V} / \mathrm{K}$, respectively. The drift of the ADC is shown in Fig. 21, as an example.

\section{Trigger delay unit (TDU)}

The TDU [7] module generates delayed trigger pulses to activate accelerator instruments, such as a klystron modulator and the DAQ system. This output pulse is triggered by its own input pulses transmitted from the MTU. The hardware configuration of the VME-TDU, as shown in Fig. 22, is almost the same as that of the test accelerator. The time jitter and thermal time drift of the TDU are less than $700 \mathrm{fs}$ in rms and about $2 \mathrm{fs} / \mathrm{K}$ [7], respectively.

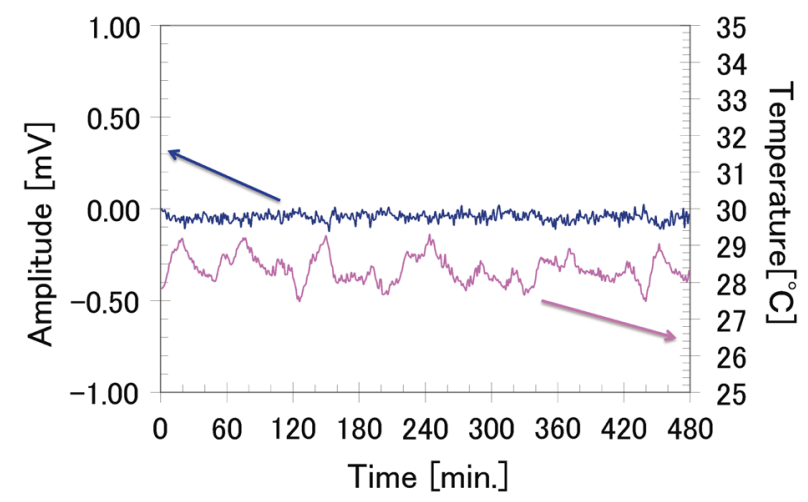

FIG. 21. Voltage conversion drift of the ADC and the environmental temperature variation, as a function of time. The thermal voltage drift of the ADC output is less than $2 \times 10^{-4} \mathrm{~V} / \mathrm{K}$.

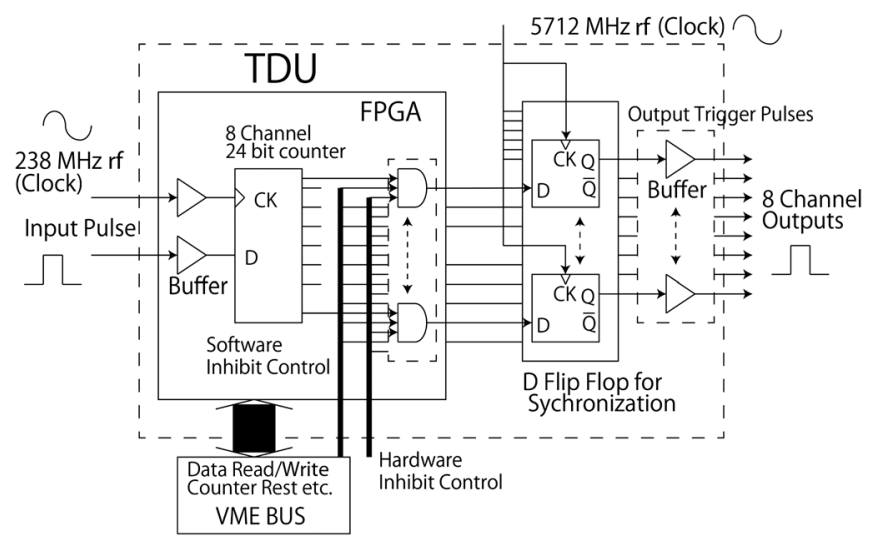

FIG. 22. Hardware configuration of the VME-TDU. The internal FPGA synchronized to a $238 \mathrm{MHz}$ rf signal has eight 24-bit counters for time delay and a 32-bit master trigger (input pulse) counter. The output pulses from the counters are resynchronized to a $5712 \mathrm{MHz}$ rf signal at the output stage for realizing a low temporal jitter.

However, the operational functions on a FPGA of the TDU are modified from that of the SCSS version so as to enhance timing control ability. These functions are described in Table III. The major modified features of the functions are as follows: One is an internal counter, which gives a time stamp for the synchronized data acquisition of a SACLA control system [28]; the next is the other counter at each output channel, which divides the input pulse frequency to individually control the repetition cycle of the klystron modulator.

\section{Klystron-driving amplifier}

Three kinds of klystron driver amplifiers operated at frequencies of 5712 ( $C$-band), 2856 ( $S$-band), and $1428 \mathrm{MHz}$ ( $L$-band) were developed for SACLA. All of the amplifiers have almost the same specifications and

TABLE III. Fundamental operational functions of the VMETDU. The functions from numbers 1 to 6 are the original functions implemented when the SCSS test accelerator was constructed; 7 and 8 are newly implemented for SACLA.

\begin{tabular}{llc}
\hline \hline 1 & Input number & 1 \\
2 & Output number & 8 \\
3 & Delay counter & 24 bits $\times 8$ channel \\
4 & $\begin{array}{c}\text { Synchronized frequency } \\
\text { of the delay counter }\end{array}$ & $238 \mathrm{MHz}$ \\
5 & $\begin{array}{c}\text { Final output synchronization } \\
\text { frequency }\end{array}$ & $5712 \mathrm{MHz}$ \\
6 & $\begin{array}{c}\text { Output enable and } \\
\text { disable function }\end{array}$ & $\begin{array}{c}\text { By software from VME } \\
\text { and hardware from } \\
\text { front panel connector }\end{array}$ \\
7 & $\begin{array}{l}\text { Counter for the input pulse } \\
8\end{array}$ & $\begin{array}{c}\text { Dividing counter for input pulse } \times 1 \text { channel } \\
16 \text { bits } \times 8 \text { channel }\end{array}$ \\
& at each output & \\
\hline \hline
\end{tabular}


configuration without their frequencies, output power values, and pulse widths. These specifications, tabulated in Table IV, are the nominal achieved performance of the amplifiers. As an example, the $C$-band amplifier has an output power of $500 \mathrm{~W}$ with an rf pulse width of $2.5 \mu \mathrm{s}$ at $5712 \mathrm{MHz}$. These amplifiers employ $A B$-class amplification, respectively. Figure 23 shows a block diagram of the $C$-band amplifier, including two amplifier stages, which comprise the $35 \mathrm{~W}$ first driving stage amplifier module and $240 \mathrm{~W}$ four final stage amplifier modules. The major difference between the SCSS's and SACLA's amplifiers is to reduce power consumption by applying pulsed biasvoltage control to field-effect transistors (FETs) as rf amplification devices.

The bias-voltage duration is minimally reduced and the requisite-minimum pulse width of the bias voltage is added to the FETs in order to generate a necessary rf pulse width for driving the klystrons. In our case, the applied biasvoltage pulse width is about $100 \mu \mathrm{s}$ for a $2.5 \mu \mathrm{s}$ rf pulse. Figure 24 depicts the output rf wave forms of the $C$-band klystron driving amplifier. The wave forms are the rf amplitude and the phase shift, as an example, and express an rf amplitude value of $500 \mathrm{~W}$ and a phase shift value of about $0.7^{\circ}$ for the amplitude pulse flattop without a rise time part of $1 \mu \mathrm{s}$. The amplitude and phase jitters are less than $0.1 \%$ in rms and $0.5^{\circ}$ in rms, respectively, and their rf amplitude and phase drifts are also less than $0.05 \%$ (P-P) and $0.1^{\circ}(\mathrm{P}-\mathrm{P})$ with a surrounding temperature variation of $0.1 \mathrm{~K}$. The measurement accuracy of the amplitude and

TABLE IV. Specifications of the klystron driver amplifiers for $L$-band, $S$-band, and $C$-band klystrons. The specifications of the amplifiers are almost the same except for its frequency.

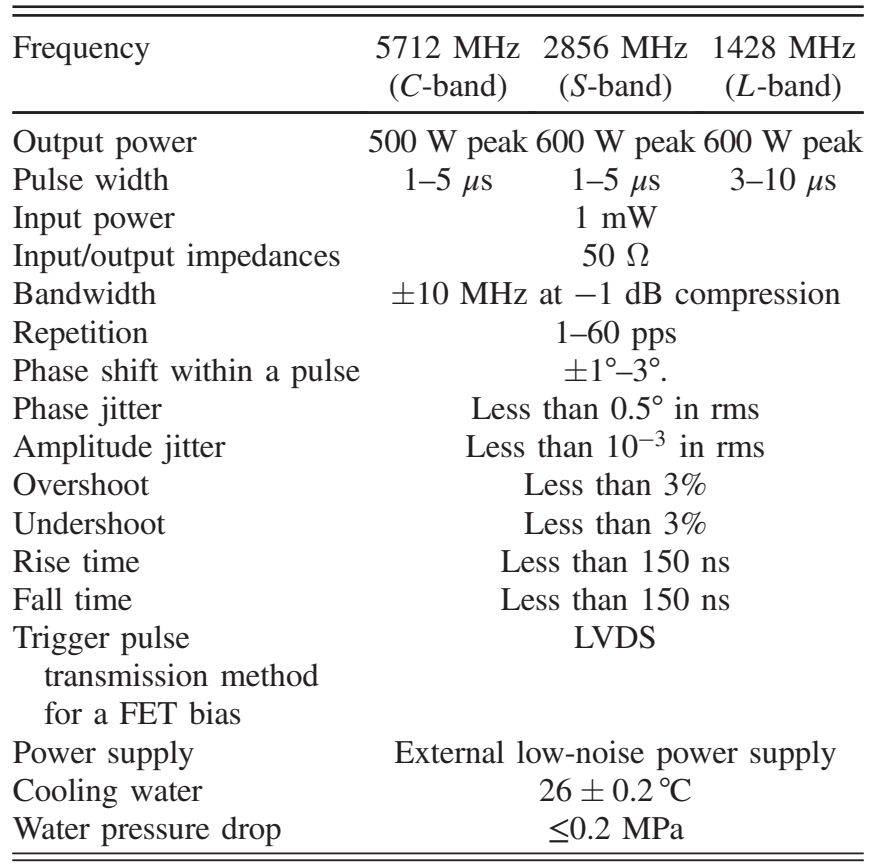

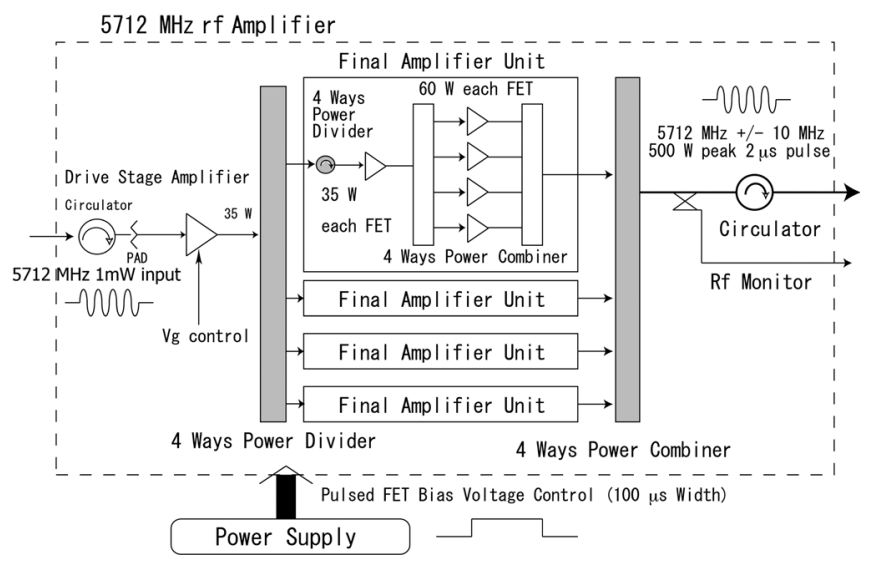

FIG. 23. Block diagram of the $C$-band amplifier. This amplifier includes two amplifier stages, which comprise the $35 \mathrm{~W}$ first drive stage amplifier module and the $240 \mathrm{~W}$ four final amplifier modules.

phase jitter values are limited by the accuracy of an $\mathrm{rf}$ network analyzer.

\section{Low-noise power supply}

Low-noise signals generated with LLRF instruments and the stable rf phase and amplitude of the instruments are indispensable to secure stable electron bunch compression. The low noise and stable output voltages of a dc power supply used to drive the LLRF instruments are necessary conditions. The inherent noise level of LLRF solid-state devices, such as a noise power spectrum of several $\mathrm{nV} / \sqrt{\mathrm{Hz}}$, are sufficiently low to a noise level determined by the demanded rf phase and amplitude stabilities. Hence, we think that the noise quality of a power supply almost decides the noise levels of the LLRF instruments, if

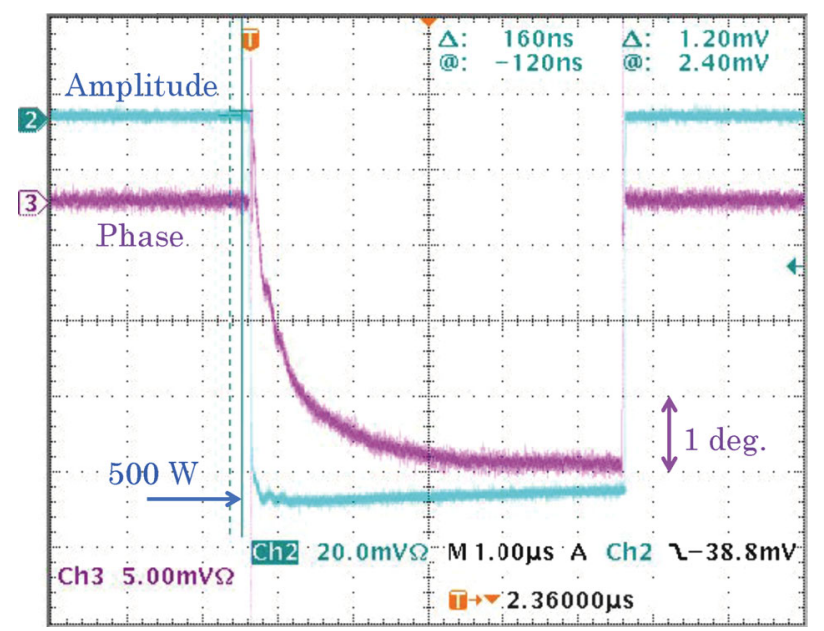

FIG. 24. Output wave forms of the $C$-band amplifier. The wave forms show an rf amplitude shift at $500 \mathrm{~W}$ and a phase shift within the pulse flattop. The phase shift for the pulse flattop is about $0.7^{\circ}$ without a rise time part of $1 \mu \mathrm{s}$. 


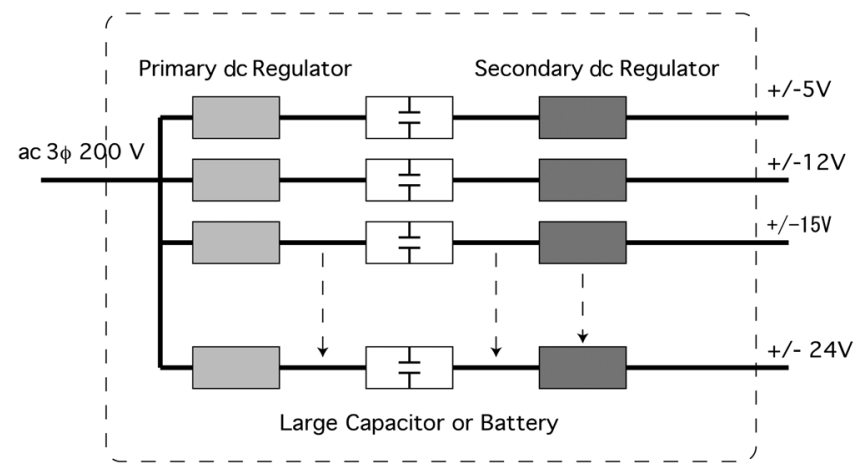

FIG. 25. Configuration of the LNPS. The LNPS uses the primary regulators, the capacitors that are like noise filters, and the secondary dc regulators. These secondary regulators supply and distribute clean dc powers having the noise level of a battery.

environmental noise components are ignored. In accordance with the above-mentioned discussion, we developed an ultralow-noise and ultrastable dc power supply (LNPS) for the LLRF system, as shown in Fig. 25. The target performance of the output noise of the LNPS is a battery, which has the cleanest power without noise. This LNPS comprises primary dropper dc power supplies and secondary dropper dc power supplies (linear dc-dc converters). Both the primary and secondary power supplies are individually and sequentially connected to achieve the battery noise quality. The circuit parameters of these primary power supplies, such as the voltage conversion ratios of ac transformers, are optimally chosen to reduce the voltage dropping losses. The function of this primary and secondary power supplies system in the figure is as follows. At first, a transformer converts the voltage of ac power to low voltage, and then diodes and capacitors rectify the power. Next, a voltage regulator, as the primary dc power supply, regulates this rectified power. The power charges very large capacitors, which have the role of a battery and act as a noise filter. After this, the charged dc power is individually fed into different kinds of the linear voltage converters (secondary dc power supplies) to make different kinds of voltages, such as $\pm 5 \mathrm{~V}$ and $\pm 12 \mathrm{~V}$, to drive the LLRF instrument, as mentioned above. Table $\mathrm{V}$ tabulates the main features of this LNPS. To mitigate any effects from the environmental noises and ac ripples, the wirings of the dc and ac lines are separated at a maximum, and the iron shields between an ac circuit and a dc circuit are installed in the enclosure of the LNPS. These separation and iron shield methods are also utilized to a component arrangement in the cage of the LLRF instrument. Figures 26 and 27 show the voltage noise level and long-term voltage stability of the LNPS, as an example, respectively. These noise and stability values are less than $-140 \mathrm{dBV}$ in a regime over a frequency of $10 \mathrm{~Hz}$ and $4 \mathrm{ppm} / \mathrm{K}$ at an output voltage of $12 \mathrm{~V}$, respectively. Their performances are sufficient for our demand.
TABLE V. Specifications of the LNPS-1.

\begin{tabular}{lc}
\hline \hline Voltage regulation method & Series regulation \\
\hline $\begin{array}{l}\text { ac input voltage/phase/ } \\
\text { frequency }\end{array}$ & $200 \mathrm{~V}, 3 \phi, 60 \mathrm{~Hz}$ \\
dc output voltage/current & $+12 \mathrm{~V}, 3.7 \mathrm{~A}$ \\
(ten outputs) & $12 \mathrm{~V}, 3.2 \mathrm{~A}$ for VME \\
& $+8 \mathrm{~V}, 12 \mathrm{~A}$ \\
& $+5 \mathrm{~V}, 24 \mathrm{~A}$ \\
& $+5 \mathrm{~V}, 60 \mathrm{~A}$ \\
& $+11 \mathrm{~V}, 1.2 \mathrm{~A}$ \\
& $+5 \mathrm{~V}, 15.2 \mathrm{~A}$ \\
& $-12 \mathrm{~V}, 1.7 \mathrm{~A}$ \\
& $-12 \mathrm{~V}, 1.0 \mathrm{~A}$ \\
Output power capacity & $810 \mathrm{~W}$ \\
Voltage stability & Below $10 \mathrm{ppm}$ \\
Voltage noise level & Below $-140 \mathrm{dBV}$ over $10 \mathrm{~Hz}$, except \\
& for a $60 \mathrm{~Hz}$ ac frequency and its \\
higher harmonic components \\
Thermal voltage drift & Below $\pm 10 \mathrm{ppm} / \mathrm{K}$ \\
Cooling method & Water cooling \\
\hline \hline
\end{tabular}

\section{Water-cooled hematothermal 19-inch enclosure}

Temperature stability is the most crucial factor to reduce characteristic drifts of the LLRF instruments, such as the rf phase and the amplitude. Hence, the LLRF instruments, as mentioned above, should be installed in a constanttemperature environment. For this reason, we developed a hematothermal 19-inch enclosure to install the instrument. There are several methods to control the inner temperature of an enclosure, such as water cooling, the Peltier effect, and the ordinal air conditioner using the Joule-Thomson effect. From these methods, we chose the water-cooling method because of costs, capacity, and controllability. Figure 28 shows the developed water-cooled 19-inch enclosures and their inner structures. The temperature stability of the enclosure within $\pm 0.1 \mathrm{~K}$ is also depicted

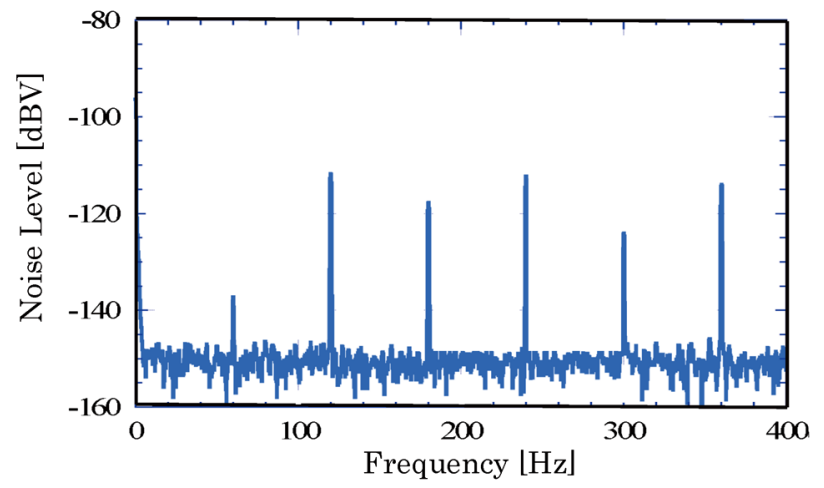

FIG. 26. Voltage noise frequency spectrum at the $12 \mathrm{~V}$ output of the LNPS. The noise level is less than $-140 \mathrm{dBV}$ in a frequency regime over $10 \mathrm{~Hz}$, except for a $60 \mathrm{~Hz}$ commercial ac power peak and its harmonics peaks. 


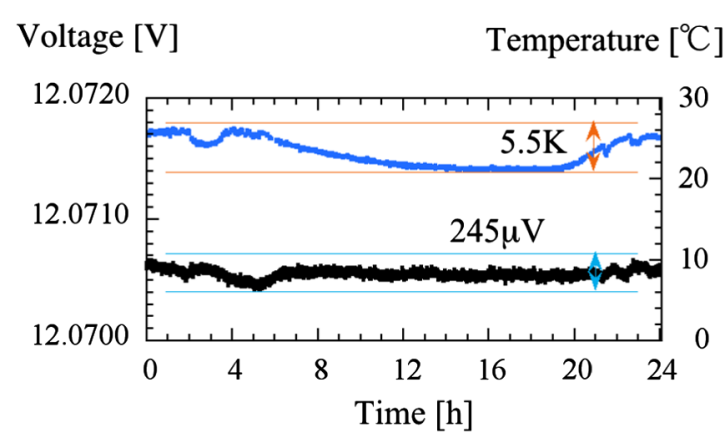

FIG. 27. Voltage stability at the $12 \mathrm{~V}$ output of the LNPS. This stability value is less than $4 \mathrm{ppm} / \mathrm{K}$, while the surrounding temperature change of the LNPS is $5.5 \mathrm{~K}$.

in Fig. 29. In the enclosure, a heat-exchange unit is placed at the left side of the enclosure, and space for installing instruments is on the right side. Circulating air in the enclosure absorbs heat generated with the LLRF instruments. Then, the air flows between the installed instruments and the heat exchanger. The heat exchanger exchanges heat from the air to the cooing water, controlled within $\pm 0.1 \mathrm{~K}$. The circulated air is directly fed from the sidewall of the instrument case to its inside. This airflow method is for mitigating the vibrations of rf cables and optical fibers placed in front of the enclosure. The individually installed instruments are also specially designed to mitigate the vibration of its inner cable due to the circulated air. This vibration directory connects to the rf phase and amplitude jitter. The x-ray laser intensity is the most sensitive to variations of the rf phases and the amplitude of the injector acceleration rf fields. The variations are also very susceptible to an environmental temperature change. Hence, the temperatures of the inner air of the injector enclosures are specially controlled to within $\pm 0.01 \mathrm{~K}$ by the previously mentioned method, as shown in Fig. 30. Highly controlled cooling water within $0.01 \mathrm{~K}$ with a

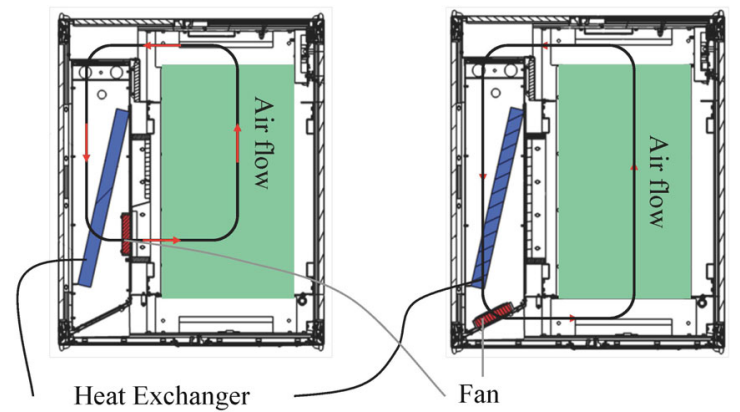

FIG. 28. Water-cooled 19-inch enclosures and its inner structures (top views). A heat-exchanger unit is located at the left side of the enclosure; space for installing instruments is on the right side. The left-hand drawing shows a sidewall cooling type, with which the circulated air is injected from its sidewall to installed instruments. The right-hand one is a front cooling type, by which the air is inputted from instrument front panels.

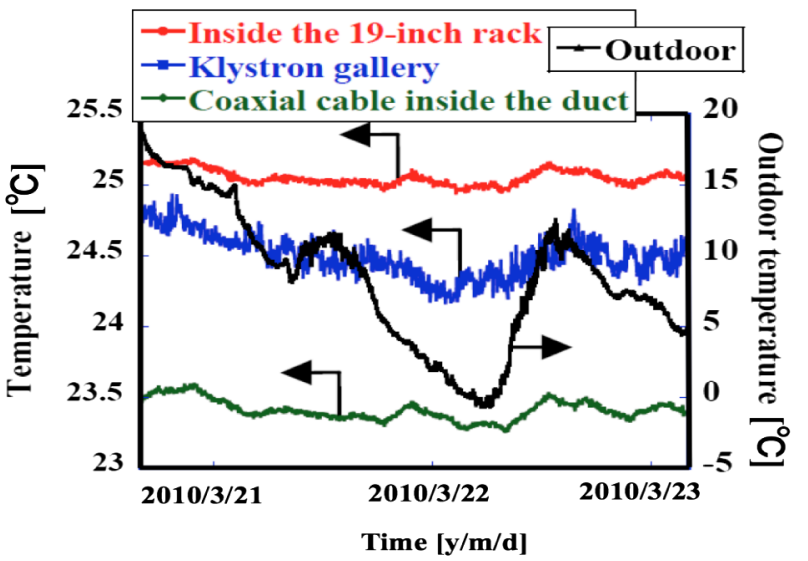

FIG. 29. Temperature stability of the water-cooled 19-inch enclosure. The most upper side line is the temperature change of the enclosure. The next line is the environmental (klystron gallery) temperature change around the enclosure. The third is an outside temperature change, and the fourth is the temperature stability of a coaxial cable duct, similarly to the optical-fiber duct, as shown in Fig. 5. The inner temperatures of the enclosure and the cable duct are controlled to within $\pm 0.1 \mathrm{~K}$.

programmable logic-controller-based temperature controller [29] is only used around the injector part. The other part of the SACLA uses cooling water controlled within a temperature of $\pm 0.1 \mathrm{~K}$. The utility system of SACLA supplies this water.

\section{0. rf phase and amplitude feedback control at cavities}

In order to reduce the phase and amplitude drifts of the rf cavities, feedback control processes are applied to the LLRF system. Both the phase and amplitude of the rf cavities in the upstream part of the injector are controlled, whereas only the rf phases of accelerating guides driven with the high-power klystrons are controlled. The installed places of these accelerating guides are remaining parts without the upstream part. The reason why we control only

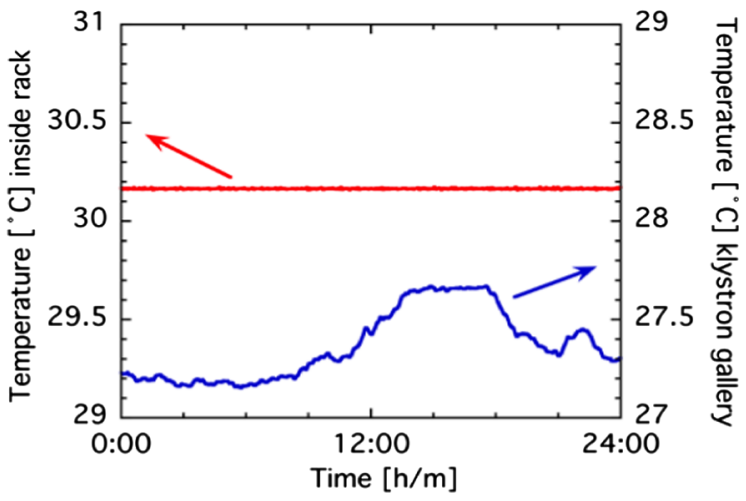

FIG. 30. Stability of the highly temperature-controlled enclose in the injector (upper line). The temperature stability value is within $\pm 0.01 \mathrm{~K}$. The line below shows the environmental temperature of the klystron gallery. 


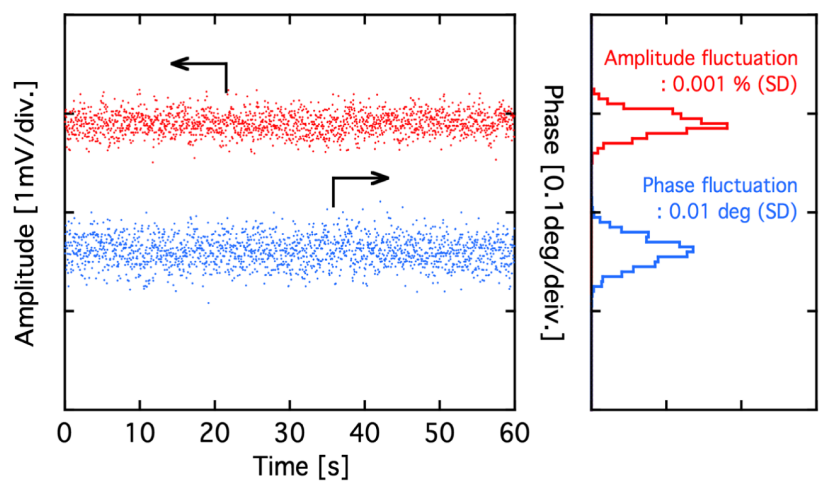

FIG. 31. rf phase and amplitude control performance of the $238 \mathrm{MHz}$ SHB. The phase and amplitude are controlled by the PID method, referred to a temporal reference of the $238 \mathrm{MHz}$ rf signal transmitted by the timing and LLRF system. The phase stability is $0.01^{\circ}$ in $\mathrm{SD}$ and the amplitude stability is $1 \times 10^{-5}$ in $\mathrm{SD}$, respectively.

the rf phases of the klystron-driven parts is that the klystron has a saturation characteristic between its input and output, as $C$-class amplification. Its output power is insensitive to any input power change in the case of $C$-class amplification. Both the phase and amplitude controls are used for reducing long-term drifts of the rf phase and amplitude in a low-frequency regime below $1 \mathrm{~Hz}$ because of the accelerator repetition frequency being $60 \mathrm{~Hz}$ or below. The control algorithm of the rf phase and amplitude represents the proportional, integral, and differential (PID) method. The basic concept of this control algorithm was confirmed in advance at the SCSS test accelerator [7]. Figure 31 shows the rf phase and amplitude control performance of the $238 \mathrm{MHz} \mathrm{SHB}$, as an example. In the case of the figure, the phase stability is $0.01^{\circ}$ in standard deviation (SD), and the amplitude stability is $1 \times 10^{-5}$ in SD. Table VI

TABLE VI. Measured rf phase and amplitude stabilities at the individual acceleration cavities. The numbers described in the parentheses are the designed values given in Table I. These data of the cavity phase and amplitude stabilities were measured for $60 \mathrm{~s}$ at an acceleration repletion frequency of $30 \mathrm{pps}$. The total number of points of data used for this measurement was 3600 ; these measured data were 40 point averaged values within onewave forms.

\begin{tabular}{lll}
\hline \hline Cavity & $\delta V / V(\%$ in SD) & $\delta \phi($ deg. in SD) \\
\hline 238 MHz SHB & $\pm 0.012( \pm 0.01)$ & $\pm 0.008( \pm 0.01)$ \\
476 MHz booster & $\pm 0.009( \pm 0.01)$ & $\pm 0.007( \pm 0.02)$ \\
$L$-band correction cavity & $\pm 0.05( \pm 0.03)$ & $\pm 0.03( \pm 0.06)$ \\
$L$-band APS cavity & $\pm 0.055( \pm 0.01)$ & $\pm 0.034( \pm 0.06)$ \\
$C$-band correction cavity & $\pm 0.135( \pm 0.1)$ & $\pm 0.084( \pm 0.06)$ \\
$S$-band accelerator & $\pm 0.012( \pm 0.01)$ & $\pm 0.048( \pm 0.1)$ \\
$C$-band accelerator & $\pm 0.024( \pm 0.01)$ & $\pm 0.042( \pm 0.2)$ \\
$\quad$ (upstream) & & \\
104 $C$-band accelerator & $\pm 0.075( \pm 0.01)$ & $\pm 0.050( \pm 0.5)$ \\
$\quad$ (downstream) & & \\
\hline \hline
\end{tabular}

describes the rf phase and the amplitude stabilities at the individual cavities of the SACLA accelerator. This PID method controls the phases and/or amplitudes of these cavities described in Table VI. Both the rf phase and amplitude are controlled at the $238 \mathrm{MHz}$, the $476 \mathrm{MHz}$, and the $C$-band correction cavities, which are driven by medium power $(50-100 \mathrm{~kW})$ pulsed-rf solid-state amplifiers. These data of the cavity phase and amplitude stabilities in the table were measured for $60 \mathrm{~s}$ at an acceleration repletion frequency of 30 pps. These stabilities are almost sufficient for our required values.

\section{PERFORMANCE OF THE TIMING AND LLRF SYSTEM}

\section{A. Short-term temporal performances}

The short-term temporal performance of the timing and LLRF system for SACLA is very important, since it determines the short-term $\mathrm{x}$-ray laser performance like intensity fluctuation. We evaluated this performance by the following three methods. One was a noise performance measurement for the system. The next was observing a temporal electron beam jitter with a cavity-type beamposition monitor (BPM) at the machine end of the SACLA. The validity of the temporal jitter measurement using the BPM was already demonstrated at the SCSS test accelerator [7]. The third was to measure the beam jitter observed with an rf beam deflector (RFDEF) [30]. Direct observation of the temporal jitter of the system is impossible by the present technology, such as using an oscilloscope. Therefore, these measurement results are indirect evidence to express the short-term temporal stability of our timing and LLRF system.

The signal source analyzer measured the increase of noise generated with MOSC along the instruments serialconnection chain of the timing and LLRF system. This noise increase directly connects to increase the electronbeam temporal jitter. It was measured at the IQ modulator output and expresses the noise generated by the instruments chain for the MOSC at the master terminal and the IQ modulator at the end terminal. The increase also includes noise generated by the optical reference signal transmission system. Figure 14 [7] shows the noise frequency spectra at the MOSC output and the output of the IQ modulator. The increase of the noise level between the MOSC output and the IQ modulator output is shown in a circle on the figure. This increased noise corresponds to a temporal jitter of $7 \mathrm{fs}$ in rms. The measured noises values of the individual instruments are summarized in Table VII. Figure 31 and Table VI also express indirect facts of the overall short-term rf phase and amplitude performances of our timing and LLRF system. These short-term performances are sufficient for our demands, described in the introduction.

The temporal beam jitter at the end part of the undulator beam line, BL3, was observed with a BPM system [7], as 
TABLE VII. Summary of the noise values and stability values for $8 \mathrm{~h}$ (typical) in the timing and LLRF instruments. The noise, amplitude, and phase values were measured at a frequency of $5712 \mathrm{MHz}$, if a frequency parameter is not defined in the table.

\begin{tabular}{|c|c|c|}
\hline Instrument & Noise (designed) and/or resolution@ @ $5712 \mathrm{MHz}$ & Stability (designed) @ $5712 \mathrm{MHz}$ \\
\hline $\begin{array}{l}\text { MOSC } P, \text { rf power; } \\
f, \text { rf frequency; } \\
\phi, \text { rf phase }\end{array}$ & $\begin{array}{c}\delta P / P=-150.6 \mathrm{dBc} / \mathrm{Hz} \\
(<-140 \mathrm{dBc} / \mathrm{Hz}) \text { at } 1 \mathrm{MHz} \\
\text { offset point }(\mathrm{SSB}), \text { rf noise }\end{array}$ & $\begin{array}{c}\delta f / f=10^{-10} / \text { day } \\
\left(<10^{-10} / \text { day }\right) \\
\text { Frequency stability } \\
\delta P / P=0.25 \% / \mathrm{K} \\
(<0.5 \% / \mathrm{K}) \\
\delta \phi=0.99^{\circ} / \mathrm{K} \\
\left(<0.1^{\circ} / \mathrm{K}\right)\end{array}$ \\
\hline $\begin{array}{l}\text { Optical transmitters } \\
\text { (OPT TXU-A and C) }\end{array}$ & $\begin{array}{c}\delta P / P=-150.0 \mathrm{dBc} / \mathrm{Hz}(<-140 \mathrm{dBc} / \mathrm{Hz}) \\
1 \mathrm{MHz}(\mathrm{SSB}), \text { rf noise }\end{array}$ & $\begin{array}{c}\delta P / P=0.12 \% / \mathrm{K} \\
\quad(<0.5 \% / \mathrm{K}) \\
\delta \phi=2.41^{\circ} / \mathrm{K} \\
\quad\left(<1^{\circ} / \mathrm{K}\right) \\
\text { Thermal rf stability }\end{array}$ \\
\hline $\begin{array}{l}\text { Optical-fiber-length control } \\
\text { (OFLCT) performance }\end{array}$ & Not assigned & $\begin{array}{l}\quad 0.3 \mathrm{fs}(<0.1 \mu \mathrm{m}) \\
\text { For } 10 \mathrm{~h} \text { and within } \delta T=0.5 \mathrm{~K}\end{array}$ \\
\hline $\begin{array}{l}\text { Optical transmitter for } \\
\text { OFLCT (OPT TX-MON) }\end{array}$ & Not measured & Not measured \\
\hline $\begin{array}{l}\text { Optical interferometer } \\
\text { for OFLCT (OPT-IF) }\end{array}$ & Not measured & Not measured \\
\hline Optical monitor for & $\delta P / P=-146.6 \mathrm{dBc} / \mathrm{Hz}(<-140 \mathrm{dBc} / \mathrm{Hz})$ & $\delta P / P=2.8 \times 10^{-3}$ in $\mathrm{rms}$ \\
\hline OFLCT (OPT-MON) & $\begin{array}{c}1 \mathrm{MHz}(\mathrm{SSB}), \mathrm{rf} \text { noise } \\
\text { Short-term amplitude stability } \delta P / P=2.88 \times 10^{-4} \\
\text { in rms short-term amplitude stability } \\
\delta \phi / \phi=0.012^{\circ} \text { in rms }\end{array}$ & $\delta \phi / \phi<0.151^{\circ}(\mathrm{P}-\mathrm{P})$ for $8 \mathrm{~h}, \delta T=0.4 \mathrm{~K}$ \\
\hline rf distributer (RF-DIS) & $\begin{array}{c}\delta P / P=-150.0 \mathrm{dBc} / \mathrm{Hz}(<-140 \mathrm{dBc} / \mathrm{Hz}) \\
1 \mathrm{MHz}(\mathrm{SSB}), \mathrm{rf} \text { noise short-term amplitude stability, } \\
\delta P / P=5.74 \times 10^{-4} \text { in rms short-term phase } \\
\text { stability, } \\
\delta \phi / \phi=0.315^{\circ}\end{array}$ & $\begin{array}{c}\delta P / P=1.47 \times 10^{-4} \\
\delta \phi / \phi=0.0783^{\circ} \text { at } \pm 0.1 \mathrm{~K} \text { for } 2 \mathrm{~h}\end{array}$ \\
\hline IQ modulator & $\begin{array}{c}\delta P / P<-145.0 \mathrm{dBc} / \mathrm{Hz}(<-130 \mathrm{dBc} / \mathrm{Hz}) \text { at } \\
1 \mathrm{MHz}(\mathrm{SSB}), \mathrm{rf} \text { noise } \sim 10^{-3}, \text { rf amplitude } \\
\text { resolution } \pm 0.1^{\circ}, \text { rf phase resolution }\end{array}$ & $\begin{array}{c}\sim 10^{-3} / \mathrm{K} \text { rf amplitude } \\
\sim 0.1^{\circ} / \mathrm{K} \text { rf phase Thermal rf stabilities }\end{array}$ \\
\hline IQ demodulator for $5712 \mathrm{MHz}$ & $\begin{array}{c}\delta P / P<-130.0 \mathrm{dBc} / \mathrm{Hz}(<-130.0 \mathrm{dBc} / \mathrm{Hz}) \\
\text { at } 1 \mathrm{MHz}(\mathrm{SSB}) \text { base-band noise } \delta P / P \sim 10^{-3} \\
\text { demodulated amplitude resolution } \pm 0.1^{\circ} \\
\text { demodulated phase resolution }\end{array}$ & $\begin{array}{c}\sim 10^{-3} / \mathrm{K} \text { base-band amplitude } \sim 0.1^{\circ} / \mathrm{K} \\
\text { base-band phase thermal } \\
\text { base-band stabilities }\end{array}$ \\
\hline 16-bit VME $238 \mathrm{MHz}$ ADC & 12.5 bit, effective ( $>12$ bit) rf amplitude resolution & $\begin{array}{c}<10^{-3} / \mathrm{K}\left(<10^{-3} / \mathrm{K}\right), \text { Thermal } \\
\text { amplitude stability }\end{array}$ \\
\hline 16-bit VME $238 \mathrm{MHz}$ DAC & 12.5 bit, effective ( $>12$ bit) rf amplitude resolution & $\begin{array}{c}<10^{-3} / \mathrm{K}\left(<10^{-3} / \mathrm{K}\right), \text { Thermal } \\
\text { amplitude stability }\end{array}$ \\
\hline $\begin{array}{l}\text { Klystron driver amplifier } \\
\text { (5712 MHz) (rf Power) }\end{array}$ & $0.1 \%$ (rms) rf amplitude noise & $\begin{array}{c}\delta P / P=10^{-3}\left(<10^{-3} \text { with } \pm 0.2 \mathrm{~K}\right) \\
\text { thermal amplitude stability }\end{array}$ \\
\hline $\begin{array}{l}\text { Klystron driver amplifier } \\
\text { (5712 MHz) (rf phase) }\end{array}$ & $0.5^{\circ}(\mathrm{rms}) \mathrm{rf}$ phase noise & $1^{\circ} / \mathrm{K}\left(<1^{\circ} / \mathrm{K}\right)$, thermal phase stability \\
\hline VME master trigger unit (MTU) & $1.6 \mathrm{ps}(\mathrm{rms})[<10 \mathrm{ps}(\mathrm{rms})]$ time jitter & Not measured \\
\hline VME trigger delay unit (TDU) & $700 \mathrm{fs}(\mathrm{rms})[<1 \mathrm{ps}(\mathrm{rms})]$ time jitter & $\sim 400 \mathrm{fs} / \mathrm{K}$, thermal jitter stability $(<1 \mathrm{ps} / \mathrm{K})$ \\
\hline $\begin{array}{l}\text { Low-noise power supply } \\
\text { (LNPS) }\end{array}$ & $-140 \mathrm{dBV} / \mathrm{Hz}$ over a frequency of $10 \mathrm{~Hz}$ & $\begin{array}{l}\text { Thermal voltage stability, } 4 \mathrm{ppm} / \mathrm{K} \\
(10 \mathrm{ppm} / \mathrm{K})\end{array}$ \\
\hline $\begin{array}{l}\text { Water-cooled hematothermal } \\
\text { 19-inch enclose }\end{array}$ & NA & $\begin{array}{l} \pm 0.01 \mathrm{~K}( \pm 0.01 \mathrm{~K}) \text { for the injector } \\
\quad \pm 0.1 \mathrm{~K}( \pm 0.2 \mathrm{~K}) \text { for the others }\end{array}$ \\
\hline Water-cooled optical-fiber duct & NA & $\pm 0.1 \mathrm{~K}( \pm 0.2 \mathrm{~K})$ \\
\hline
\end{tabular}

shown in Fig. 32. The BPM uses a $4760 \mathrm{MHz}$ detection frequency in order to prevent any effect due to dark current generated by $C$-band acceleration guides operated at $5712 \mathrm{MHz}$. The temporal jitter corresponds to the phase fluctuation between a reference rf signal distributed by the timing and LLRF system and an induced rf field due to the electron beams into the intensity detection cavity of the BPM. This intensity detection cavity is used for obtaining a 


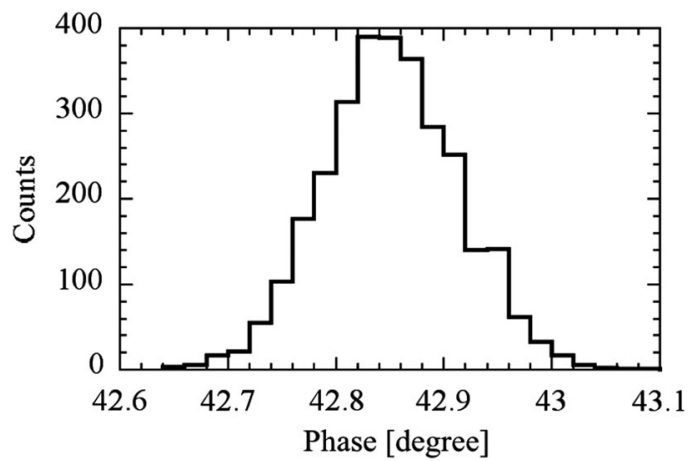

FIG. 32. Beam arrival time jitter measured with the BPM at the end part of the undulator beam line BL3. The short-term temporal jitter is less than $0.14^{\circ}$ at $4760 \mathrm{MHz}$, corresponding to $81.7 \mathrm{fs}$ in FWHM. The total number of beam shots is 3010 .

factor to normalize the beam-position signals. A $4760 \mathrm{MHz}$ IQ demodulator, whose relative phase measurement accuracy is less than $0.1^{\circ}$, measures the temporal jitter of the electron beam at the intensity detection cavity. From the results of the observation, the short-term temporal jitter is less than 81.7 fs in FWHM.

The beam arrival time jitter [26] was measured with the RFDEF, and the jitter measurement result is described in Fig. 33. In this case, the beam arrival time jitter is $13.6 \mathrm{fs}$ in rms with the conditions that the beam energy was $1.45 \mathrm{GeV}$, the beam charge was about $0.3 \mathrm{nC}$, and the beam repetition cycle was 1 pps. This temporal measurement resolution is about $10 \mathrm{fs}$, which is also limited by a natural beam size on a screen monitor conducted by a normalized emittance of about $1.1 \mathrm{~mm}$ mrad and a beta function at the screen monitor of $60 \mathrm{~m}$. This measurement accuracy shows evidence of how the low-level rf system for SACLA is stable in the short term.

\section{B. Long-term temporal performance}

The long-term performance (drift) of the timing and LLRF instruments was evaluated under practical operation,

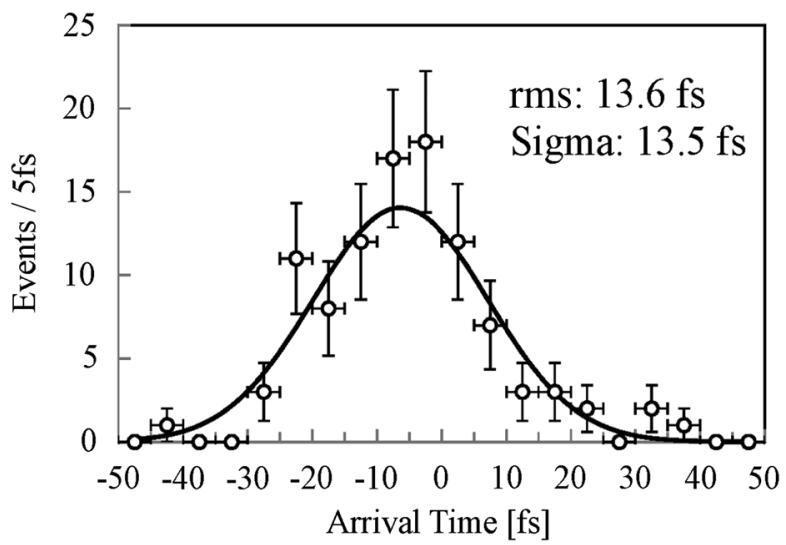

FIG. 33. Beam arrival time jitter measured with the RFDEF at a location just after BC-3. The temporal jitter is $13.6 \mathrm{fs}$ in rms. The total number of beam shots is 100 .

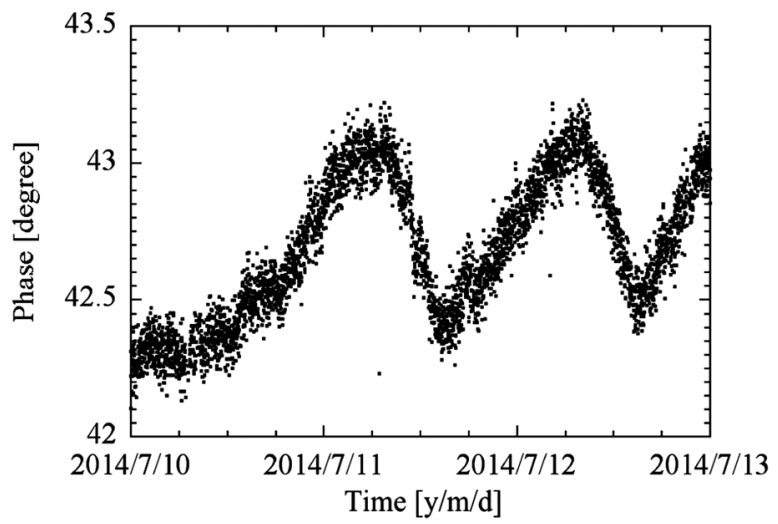

FIG. 34. Beam arrival time drift at the end part of BL-3. The drift is less than about $1^{\circ}$ for 3 days, which corresponds to a temporal drift of less than $580 \mathrm{fs}$. These data include the phase drift of the BPM detection circuit.

such as for user experiments. Table VII gives the thermal drift performances of the individual instruments. These drift performance are sufficient for our required values, as mentioned previously. The long-term performance of the timing and LLRF system, including the optical-fiber-length control performance, was also indirectly checked in order to measure the temporal drift of the beam arrival time at the end of the BL3, as mentioned above. The temporal drift is depicted in Fig. 34 and is about $1^{\circ}$ at $4760 \mathrm{MHz}$, corresponding to 580 fs for 3 days.

In the current SACLA's machine stability condition, if the operator of SACLA trims the rf phases of the injector cavities in about once every $4 \mathrm{~h}$, the lasing intensity can be maintained within about $10 \%$ in rms for $8 \mathrm{~h}$, as shown in Fig. 35. This lasing stability is also established by feedback control for rfs and beam bunch widths by coherent synchrotron radiation monitors [26,31] at the BCs.

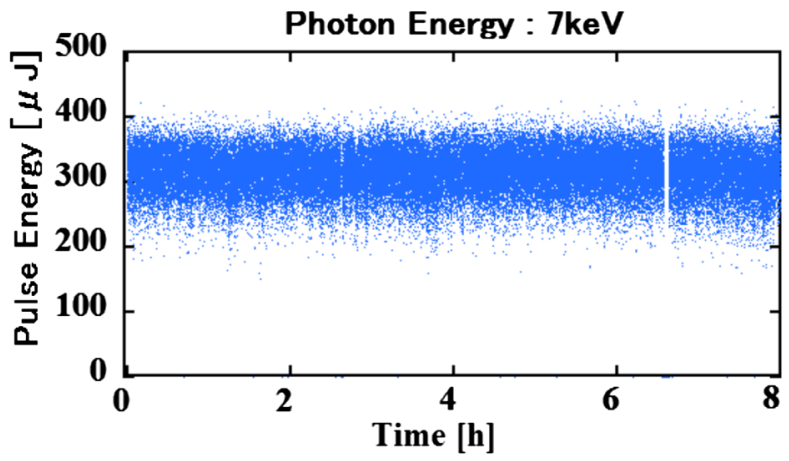

FIG. 35. X-ray laser intensity drift of SACLA for $8 \mathrm{~h}$. The photon energy was $7 \mathrm{keV}$, and its pulse repetition was 30 pps. This lasing stability is established by minor operator trimming of the rf phase at the injector cavities at about once every $4 \mathrm{~h}$ and feedback control loops for rfs and beam bunch widths by coherent synchrotron radiation monitors at the BCs. 


\section{SUMMARY}

A timing and LLRF system for XFEL was developed, installed into SACLA, and tested during machine operation. Optical-fiber reference signal transmission using PSOFs with optical-fiber-length control by the Michelson interferometry method was developed to be adapted to large machine size and the required temporal drift stability. The optical signal transmission system successfully distributes $5712 \mathrm{MHz}$ and several other rf signals for klystrons, diagnostics, and experimental users with the demanded performance.

The optical-fiber-length control system regulates the length of the PSOF installed in the water-cooled hematothermal duct up to a length change of less than $1 \mu \mathrm{m}$, corresponding to $3 \mathrm{fs}$ for about $1 \mathrm{~km}$. This performance is achieved within an optical-fiber-length control loop. In order to control the rf phase and amplitude of an acceleration structure, a LLRF system based on an IQ modulator and a demodulator was designed and constructed. This modulator allows mitigating the cross-term effect between the rf phase and the amplitude by cross-term reduction calibration. After the calibration, the output rf phase and amplitude variations of the IQ modulator are reduced from $5^{\circ}$ and $1 \%$ to within $1^{\circ}$ and $1 \%$ along $360^{\circ}$ control phase changes, respectively. The ultralow-noise level of our system allows for a short-term temporal stability of a femtoseconds regime of less than 50 fs to be the demanded extremely tight tolerance. For example, the short-term temporal stability of the system is less than $13.6 \mathrm{fs}$ in rms, which was indirectly observed by beam arrival time jitter measurements using the RFDEF. The long-term temporal stability is less than $600 \mathrm{fs}$, which was measured by the beam arrival time at the BPM. We consider that this parameter drift is caused by a change in the environmental condition, such as the temperature and humidity. There are some evident correlation data between the cavity rf phases and the environmental condition change, especially concerning the humidity. The details of this fact will be reported in another paper. We think that the long-term stability is not perfectly sufficient for the temporal demand because of the cyclic operator trimming at once every $4 \mathrm{~h}$. However, it is still acceptable for SACLA's user operation.

Using the following methods was a strong driving force to achieve these tight tolerance requirements. These employed methods are low-noise microwave devices, an ultrastable temperature environment around the timing and LLRF instruments within $10 \mathrm{mK}$, realized by water-cooled 19-inch enclosures and optical-fiber ducts, and ultralownoise power supplies to drive the instruments. The system works well for the machine operation of SACLA and shows almost sufficient performance, such as rf phase and amplitude stability, to our demanded short-term temporal stability of $50 \mathrm{fs}$ in rms. One indirect evidence to secure the temporal stability of the system is the measured beam temporal jitter of $13.6 \mathrm{fs}$ in rms with the RFDEF. This value is almost below our simulated number and meets our demanded tolerance to stably generate an x-ray laser. As a result, by using our developed timing and LLRF system, SACLA constantly lases for user experiments.

\section{ACKNOWLEDGMENTS}

The authors thank the members of the XFEL/SPring- 8 construction group for their help to our study and instrument development. The authors also appreciate the constructing work of the timing and LLRF system by the members of Mitsubishi Electric-tokki system Co., Ltd.

[1] E. L. Saldin, E. A. Schneidmiller, and M. V. Yurkov, The Physics of Free Electron Laser (Springer, New York, 2000), p. 52.

[2] SCSS X-FEL Conceptual Design Report, edited by T. Tanaka and T. Shintake (RIKEN Harima Institute, Japan, 2005).

[3] T. Ishikawa et al., Nat. Photonics 6, 540 (2012).

[4] J.C. Slater, Microwave Electronics (Van Nostrand, Princeton, 1959), pp. 222-232 and 268.

[5] M. Dohlus, T. Limberg, and P. Emma, in Electron Bunch Length Compression, edited by I.S. Ko, ICFA Beam Dynamic Letters No. 38 (2005), Chap. 4.1, pp. 15-23; http://www-bd.fnal.gov/icfabd/news.html.

[6] H. Tanaka, T. Hara, and K. Togawa, Proc. of the 4th annual meeting of PASJ (Particle Accelerator Society of Japan, Wako, 2007), p. 613, in Japanese.

[7] Y. Otake, T. Ohsima, N. Hosoda, H. Maesaka, T. Fukui, M. Kitamura, and T. Shinatale, Nucl. Instrum. Methods Phys. Res., Sect. A 696, 151 (2012).

[8] T. Shintake et al., Nat. Photonics 2, 555 (2008).

[9] P. Horowitz and W. Hill, The Art of Electronics (Cambridge University Press, Cambridge, England, 1994), p. 727.

[10] S. Tanaka, technical report of Sumitomo Electric Ind. Ltd., 1989.

[11] N. Hosoda, H. Maesaka, S. Matsubara, T. Ohshima, K. Tamasaku, and Y. Otake, Proceedings of the International Particle Accelerator Conference, Kyoto, Japan (ICR, Kyoto, 2010), p. 2191.

[12] K. Thyagarajan and A. Ghatak, Fiber Optic Essentials (Wiley-interscience, New York, 2007), p. 193.

[13] M. Musha, Y. Sato, K. Nakagawa, K. Ueda, A. Ueda, and M. Ishiguro, Appl. Phys. B 82, 555 (2006).

[14] Y. Otake, Proceedings of 33rd International Free-electron Laser Conference, Shanghai, China (SINAP, Shanghai, 2011), p. 633.

[15] P. Hariharan, Optical Interferometry, 2nd ed. (Elsevier, New York, 2003), Vol. 26.

[16] K. Thyagarajan and A. Ghatak, Fiber Optic Essentials (Wiley-interscience, New York, 2007), p. 200.

[17] K. Thyagarajan and A. Ghatak, Fiber Optic Essentials (Wiley-interscience, New York, 2007), p. 125.

[18] D. Meschede, Optics, Light and Lasers (Wiley-vch, New York, 2007), p. 285. 
[19] P. Hariharan, Optical Interferometry, 2nd ed. (Elsevier, New York, 2003), p. 24.

[20] D. A. Jackson, R. Priest, A. Dandridge, and B. Tveten, Appl. Opt. 19, 2926 (1980).

[21] Catalog of the signal source analyzer (E5052B), Agilent technology, 2014.

[22] Recent Progress in Optical Fiber Research, edited by M. Yasin (InTEC, Janeza, Croatia, 2012), Chap. 6.4, in-Tech pp. 144-148.

[23] P. Horowitz and W. Hill, The Art of Electronics (Cambridge University Press, Cambridge, England, 1994), pp. 885 and 1038 .

[24] Catalog of Hittite Microwave Corporation, 2014.

[25] T. Ohshima, H. Maesaka, N Hosoda, S. Matsubara, and Y. Otake, Proceedings of the 12th International Conference on Accelerator and Large Experimental Physics Control Systems, Kobe, Japan (SPring-8, Japan, 2009), p. 453.

[26] Y. Otake, H. Maesaka, S. Matsubara, S. Inoue, K. Yanagida, H. Ego, C. Kondo, T. Sakurai, T. Matsumoto, and H. Tomizawa, Phys. Rev. Accel. Beams 16, 042802 (2013).

[27] Z. D. Farkas, H. A. Hogg, G. A. Loew, and P. B. Wilson, Report No. SLAC-PUB-1561, 1975.

[28] M. Yamaga, Y. Furukawa, T. Hirono, M. Ishii, T. Masuda, T. Ohata, R. Tanaka, and A. Yamashita, Proceedings of the 12th International Conference on Accelerator and Large Experimental Physics Control Systems, Kobe, Japan (SPring-8, Japan, 2009), p. 69.

[29] T. Asaka, T. Hasegawa, H. Maesaka, Y. Otake, and K. Togawa, Proceedings of 27th Linear Accelerator Conference, Geneva, Switzerland (CERN, Geneva, 2014), p. 1131.

[30] H. Ego, H. Maeska, T. Sakurai, Y. Otake, T. Hashirano, and S. Miura, Nucl. Instrum. Methods Phys. Res., Sect. A 795, 381 (2015).

[31] C. Kondo, H. Maeska, Y. Otake, S. Matsubara, T. Matsumoto, and S. Inoue, Proceedings of the 2nd International Particle Accelerator Conference, San Sebastián, Spain (EPS-AG, Spain, 2011), p. 1224. 